\title{
Common Ownership of Public Goods
}

Maija Halonen-Akatwijuka

Evagelos Pafilis

Discussion Paper 18 / 700

13 June 2018

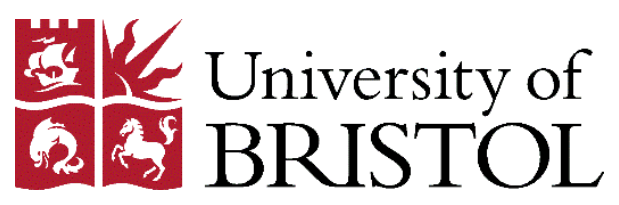

Department of Economics

University of Bristol

Priory Road Complex

Bristol BS8 1TU

United Kingdom 


\title{
Common Ownership of Public Goods ${ }^{1}$
}

\author{
Maija Halonen-Akatwijuka* and Evagelos Pafilis ${ }^{* *}$ \\ * University of Bristol \\ ** King's College London
}

May 2018

\begin{abstract}
${ }^{1}$ This paper was previously distributed under the title "Reputation and Ownership of Public Goods". We would like to thank Tim Besley, Paul Grout, Oliver Hart, Elisabetta Iossa, In-Uck Park and Armin Schmutzler for discussions and seminar audiences at Aberdeen, Bristol, King's College London, CEPR Conference on Partnerships between Governments and Private Sector in London, Contracts, Firms and Corporate Finance Conference in ParisDauphine, EARIE in Valencia and Istanbul, GW4 Game Theory Workshop in Bristol, ISNIE in Cambridge MA, PET in Galway and St Andrews Workshop in Applied Microeconomics for comments. Emails: maija.halonen@bristol.ac.uk, evagelos.pafilis@kcl.ac.uk.
\end{abstract}




\begin{abstract}
We analyze optimal ownership of public goods in a repeated game focusing on common ownership. Under common ownership an owner's access to the public good cannot be restricted by other owners. We find that under common ownership both the value of the relationship and the gain from deviation are high. Common ownership is optimal when the marginal return to maintenance investments is low consistent with the stylized facts.
\end{abstract}

JEL classification: D23, H41, L14, L33

Keywords: public goods, common pool resources, property rights, repeated games, common ownership, joint ownership 


\section{Introduction}

Ostrom (1990) challenged the argument that common pool resources (CPRs), such as grazing areas, forests and irrigation systems, are subject to overappropriation of the resource and underprovision of its maintenance. She demonstrated with case studies how common ownership has in many cases outperformed both private and public ownership - but in other cases ownership by private firms or government has been successful. She referred to repeated games in explaining how common ownership can overcome overappropriation and underprovision problems. However, also other ownership structures can work well in a repeated game. In this paper we analyze the optimal ownership structure in a repeated game focusing on the maintenance investments. We find that common ownership is optimal when the marginal return to maintenance investments is low, which is consistent with the stylized facts.

Irrigation systems in Nepal are an example of long-standing, successful common ownership arrangements (Ostrom and Gardner 1993). Communally owned irrigation systems cover $62 \%$ of the irrigated land and have outperformed systems managed by national government agency. These irrigation systems typically rely on low-tech construction techniques such as nonpermanent headworks from mud, trees and stones and unlined canals. Continual maintenance is therefore required. Every spring before the monsoon season the headworks and the canals are repaired. During the monsoon rains the canals are patrolled daily, small leaks are repaired and the community is alerted for any major damage, such as a landslide (Martin and Yoder 1988). Considerable investments are therefore required in maintaining the irrigation systems. Our focus is on finding when common ownership gives the best incentives for such repeated maintenance investments.

Property rights theory of Grossman and Hart (1986) and Hart and Moore (1990) 
- and its extension to public goods by Besley and Ghatak (2001) - is the natural framework for analyzing the optimal ownership structure. We consider two agents who make repeated maintenance investments in the public good but differ in their valuation of it. The valuation difference can arise e.g. from the size of the landholding to be irrigated. In the main model we focus on two shared ownership structures, common ownership and joint ownership, and examine single ownership - such as privatization or government ownership - in an extension.

Under common ownership, according to Ostrom and Hess (2010), an owner's access to the public good cannot be restricted by other owners (but access can be denied for non-owners). Therefore there is nothing to bargain about ex post in the static game and each agent receives his full individual valuation of the public good. Under other ownership structures the agents share the joint surplus in bargaining. Under joint ownership they split the surplus 50:50 as they have to reach a unanimous decision. Under single ownership they bargain over the non-owner's contribution to the public good. For the investment incentives in the static game, this implies that under common ownership each agent maintains the public good according to his own valuation, ignoring the benefit to the other agent. Common ownership is then dominated by ownership by the high-valuation agent in the static game.

For the repeated game, this implies that the value of an efficient relationship, as compared to one governed by a spot transaction, is high under common ownership reducing the incentive to underinvest in maintenance. However, also the gain from deviation is high under common ownership. If the high-valuation agent underinvests, the other agent observes it and, consequently, the ex post bargaining will be as in the static game. The high-valuation agent obtains maximal deviation payoff under common ownership as he gets his full valuation of the inefficiently maintained public good while under other ownership structures he has to share his high valuation in 
bargaining. ${ }^{1}$ Optimal ownership structure is then a tradeoff between increasing the value of the relationship and reducing the gain from deviation.

We find that common ownership is optimal when the marginal cost of investment is convex implying that the investment is inelastic. Then, one-shot investment is not significantly lower than the first-best investment resulting in a low value of the relationship. Guaranteeing that the relational contract is self-enforcing requires then an ownership structure that maximizes the value of the relationship and common ownership is optimal. While when the marginal cost in concave - and the investment is elastic - one-shot investment is much lower than the first-best investment and the value of the relationship is high under any ownership structure. Then, emphasis is on minimizing the gain from deviation and either joint ownership or ownership by the high-valuation agent is optimal.

In our model, convexity of marginal cost also implies that the marginal cost is high. We argue that the communally owned irrigation systems in Nepal and the Philippines can be characterized by high marginal cost, as they require considerable maintenance investments, and therefore match our results. Furthermore, if we put the curvature in the value of the investment, our result implies that the marginal value of the investment is low. This is consistent with a stylized fact of common ownership: the value of the CPR cannot be increased much by investment (Netting 1976; Ostrom 1990, p. 63). Communal grazing lands in the Swiss Alps are an example of such common ownership.

In the main model we apply trigger strategies with reversion to Nash equilibrium of the static game after any deviation. In an extension we examine renegotiation by allowing the deviator to pay a fine to the other party to restore ex post efficiency. ${ }^{2}$ This

\footnotetext{
${ }^{1}$ For the low-valuation agent there is the opposite effect but it is of a smaller magnitude.

${ }^{2}$ See e.g. Levin (2003) and Goldlücke and Kranz (2012) on repeated games with monetary transfers and e.g. Doornik (2006) and Rayo (2007) on their applications in organizations.
} 
modelling approach fits well in common property arrangements where members have to pay a fine if they do not contribute to maintenance (Ostrom 1990, p. 98). We show that, as in the private goods case of Blonski and Spagnolo (2007), renegotiation does not change the optimal ownership structure because the fine is set so that all the gains from renegotiation go to the non-defecting party. Therefore the continuation payoff for the deviating party is equal to his continuation payoff without any renegotiation, that is his payoff with trigger strategy.

Our contribution is to show when common ownership of public goods is an optimal ownership structure in a repeated game. Our work is related to the extensive literature on appropriation of CPRs in a dynamic game, e.g. Benhabib and Radner (1992) and Copeland and Taylor (2009). Benhabib and Radner (1992) show that the potential overappropriation of the $\mathrm{CPR}$ can be overcome in a dynamic game if the discount factor and the stock of the CPR are high enough. In Copeland and Taylor (2009) efficient appropriation of the CPR depends also on the strength of property rights. We complement their work by introducing maintenance investments and endogenizing the ownership structure.

Within the relational contracts literature, Baker, Gibbons and Murphy (2002) and Halonen (2002) examine ownership of private goods when the agents make repeated specific investments. They show that it depends importantly on the ownership structure whether the holdup problem can be overcome in a repeated game - similar to our analysis of public goods. (We will compare the public and private goods cases in detail in Section 6.) The structure of our model is similar to Halonen (2002) where both parties invest and the investments are observable - and different from Baker, Gibbons and Murphy (2002) where the agent makes multiple investments that are unobservable to the principal. Ramey and Watson (1997) and Halac (2015) analyze the effect of 
an upfront specific investment on a relational employment contract. In Ramey and Watson (1997) upfront investment makes the relational contract robust to negative productivity shocks by increasing the value of the relationship. In Halac (2015) the firm's upfront investment may be appropriated by the employee in ex post bargaining. The holdup problem can be eliminated by making the stochastic investment returns unobservable, potentially reducing the employee's effort. Our model differs from their setup in that both parties invest and the investments are repeated. Finally, in Harstad, Lancia and Russo (2015) countries make repeated technology investments that affect the sustainability of a relational environmental treaty. Overinvesting in green technologies, that are strategic substitutes for polluting, and underinvesting in brown technologies, that are strategic complements for polluting, reduce the gain from freeriding in emissions. In our model it is the ownership structure that is chosen to reduce incentive to freeride in maintenance investments.

Within the static property rights literature, Besley and Ghatak (2001) analyze optimal ownership of public goods and show that ownership by the high-valuation agent is optimal. We analyze their model in a repeated game. ${ }^{3}$ Rosenkranz and Schmitz (2003) and Niedermayer (2013) also compare common ownership and joint ownership although they use different terminology. ${ }^{4}$ In Rosenkranz and Schmitz (2003) joint ownership induces know-how disclosure while common ownership provides good investment incentives. Their R\&D context differs from ours by including two sequential projects and the possibility of know-how disclosure. In Niedermayer (2013) common ownership provides good incentives to invest in private goods complementary to a public good at the cost of a lower investment (by one agent) in the public good.

\footnotetext{
${ }^{3}$ Besley and Ghatak (2001) has also been extended e.g. to impure public goods (Francesconi and Muthoo 2011) and indispensable agents (Halonen-Akatwijuka 2012).

${ }^{4}$ Our joint and common ownership are equivalent to joint ownership with and without bilateral veto power in Rosenkranz and Schmitz (2003) and to standardized and open source platform in Niedermayer (2013).
} 
In our model both agents invest (only) in the public good. Finally, the incomplete contracting literature on privatization, e.g. Hart, Shleifer and Vishny (1997), and on public-private partnerships, e.g. Hart (2003) and Bennett and Iossa (2006), are related. We differ in allowing also the private providers to be value driven and modelling explicitly the public good nature of the projects.

The rest of the paper is organized as follows. Section 2 presents the static game and Section 3 analyzes the repeated game. Section 4 extends the analysis to single ownership and Section 5 examines renegotiation. Section 6 compares the results of the repeated game for public and private goods. Section 7 applies our results to CPRs and Section 8 concludes.

\section{Static game}

There are two agents, $\ell$ and $h$. Ex ante each agent makes an investment, denoted by $y_{\ell}$ and $y_{h}$, in a public good, e.g. maintaining an irrigation system. ${ }^{5}$ Ex post the agents produce the public good. The agents value the public good differently: the low-valuation agent's utility is $\theta_{\ell}\left(y_{\ell}+y_{h}\right)$ and the high-valuation agent's utility is $\theta_{h}\left(y_{\ell}+y_{h}\right)$ where $\theta_{\ell}<\theta_{h}$. Investment costs are given by $c\left(y_{i}\right)=\left(y_{i}\right)^{\gamma}$ for $i \in\{\ell, h\}$ where $\gamma>1$. The investments are observable to the agents but not verifiable to a third party.

Joint surplus equals $S=\left(\theta_{\ell}+\theta_{h}\right)\left(y_{\ell}+y_{h}\right)-c\left(y_{\ell}\right)-c\left(y_{h}\right)$. The first-best investments are then given by the following first-order conditions.

$$
\left(\theta_{\ell}+\theta_{h}\right)=c^{\prime}\left(y_{i}^{*}\right) \text { for } i \in\{\ell, h\}
$$

\footnotetext{
${ }^{5}$ In the main model the investment can be in either physical or human capital. In Section 4 the nature of the investment will matter.
} 
We denote $y^{*} \equiv y_{h}^{*}=y_{\ell}^{*}$.

Contracts are incomplete and, consequently, ex ante contracts can only be written on the ownership of the public good. In the main model we focus on two types of shared ownership, common ownership and joint ownership, and examine single ownership in Section 4. Joint ownership has often been analyzed in the property rights framework. ${ }^{6}$ Under joint ownership a unanimous agreement by the owners is required for the project to go ahead and therefore the disagreement payoffs equal zero. Common ownership is a less familiar concept in the property rights theory. ${ }^{7}$ Under common ownership, according to Ostrom and Hess (2010), an owner's access to the public good cannot be restricted by other owners although access can be denied for non-owners. Therefore each agent's utility is $\theta_{i}\left(y_{\ell}+y_{h}\right)$ for $i \in\{\ell, h\}$ even if they disagree.

The timing is the following:

Stage $1 . \quad \ell$ and $h$ contract on the ownership of the project. We analyze joint ownership and common ownership.

Stage 2. $\ell$ and $h$ invest in project-specific capital.

Stage 3. $\ell$ and $h$ bargain over the completion of the project and produce the public good.

Public goods model is appropriate for analyzing CPRs as we focus on maintenance (Ostrom 1990, p. 32). Public goods share the attribute of nonexcludability with CPRs. In our model this implies that it is not possible to exclude any owner from the improvements to the CPR. Public goods differ from CPRs in the attribute of nonrivalness. There is a large literature analyzing the appropriation of CPRs, e.g.

\footnotetext{
${ }^{6}$ For example in Besley and Ghatak (2001), Halonen (2002), Cai (2003) and Rosenkranz and Schmitz (2003).

${ }^{7}$ Rosenkranz and Schmitz (2003) call a similar arrangment joint ownership with no veto right. Niedermayer (2003) examines an open source platform where no one can be excluded from the public good. Open source and common ownership are equivalent in a 2-agent setup.
} 
Benhabib and Radner (1992) and Copeland and Taylor (2009), which we complement by introducing maintenance investments.

Under joint ownership the disagreement payoffs are zero as each agent has veto power. Therefore the agents split the ex post surplus 50:50 in Nash bargaining resulting in the following payoffs

$$
u_{i}^{J}=\frac{1}{2}\left(\theta_{\ell}+\theta_{h}\right)\left(y_{h}+y_{\ell}\right)-c\left(y_{i}\right) \quad \text { for } i \in\{\ell, h\}
$$

where superscript $J$ denotes joint ownership. Under joint ownership the investment incentives are

$$
\frac{1}{2}\left(\theta_{\ell}+\theta_{h}\right)=c^{\prime}\left(y_{i}^{J}\right)
$$

We denote $y^{J} \equiv y_{h}^{J}=y_{\ell}^{J}$. Underinvestment results as the agents share the value of investment in bargaining.

Under common ownership neither consumption of the public good nor participation in its production can be restricted for the owners. Therefore there is nothing to bargain about ex post. Consequently, the payoffs are

$$
u_{i}^{C}=\theta_{i}\left(y_{\ell}+y_{h}\right)-c\left(y_{i}\right) \quad i \in\{\ell, h\}
$$

Common ownership is denoted by superscript $C$. Optimal investments under common ownership, denoted by $y_{i}^{C}$, are given by

$$
\theta_{i}=c^{\prime}\left(y_{i}^{C}\right) \quad i \in\{\ell, h\}
$$

Each agent chooses investment according to his own valuation, ignoring the benefit of the public good to the other agent. 
(1), (3) and (5) show that $y_{\ell}^{C}<y^{J}<y_{h}^{C}<y^{*}$. Proposition 1 compares the joint surplus under common ownership and joint ownership.

Proposition 1 In the static game joint ownership dominates common ownership if and only if $\gamma>1.5$.

All the proofs are in Appendix A.1.

Under joint ownership both agents have equal, intermediate incentives while under common ownership the high-valuation agent has strong incentives and the lowvaluation agent has weak incentives. Now if $\gamma$ is high enough, two intermediate investments are more cost-effective than one high and one low investment and joint ownership is optimal.

\section{Repeated game}

In a repeated game stages 2 and 3 are played in periods $t \in\{1,2, \ldots, \infty\}$. In a subgame-perfect equilibrium (SPE) of the repeated game the agents may be able to sustain higher levels of investments than in the static game. We focus on the Pareto optimal SPE where the first-best investments are sustainable. The agents promise to make the first-best investments, $y^{*}$, and agent $h$ agrees to pay agent $\ell$ a transfer $T^{*}$ (which is not restricted to be positive). Agent $i \in\{\ell, h\}$ continues to invest $y^{*}$ and to agree to $T^{*}$ as long as the other agent keeps his promises. Any deviation triggers punishment for the rest of the game. In this Section we examine reversion to the Nash equilibrium of the stage game as punishment. In Section 5 we introduce renegotiation. 
The incentive compatibility constraints (ICs) for the agents are

$$
\begin{aligned}
& 2 \theta_{h} y^{*}-T^{*}-c\left(y^{*}\right) \geq(1-\delta) P_{h}^{d, \omega}+\delta P_{h}^{p, \omega} \\
& 2 \theta_{\ell} y^{*}+T^{*}-c\left(y^{*}\right) \geq(1-\delta) P_{\ell}^{d, \omega}+\delta P_{\ell}^{p, \omega}
\end{aligned}
$$

where $\delta \in[0,1)$ is the common discount factor. We have multiplied the payoffs by $(1-\delta)$ to express them as per-period averages. $\quad P_{i}^{d, \omega}$ is agent $i$ 's one-shot deviation payoff under ownership structure $\omega \in\{J, C\}$ and $P_{i}^{p, \omega}$ is $i$ 's payoff in the punishment path.

We first show that deviation in investment dominates deviation in transfer. If agent $i$ deviates in investment, agent $j$ observes it and starts the punishment already in the transfer stage. Therefore he will not agree to $T^{*}$ but will engage in bargaining resulting in the following payoff for agent $i$ under joint ownership ${ }^{8}$

$$
P_{i}^{d, J}=\frac{1}{2}\left(\theta_{\ell}+\theta_{h}\right)\left(y^{*}+y^{J}\right)-c\left(y^{J}\right),
$$

while under common ownership

$$
P_{i}^{d, C}=\theta_{i}\left(y^{*}+y_{i}^{C}\right)-c\left(y_{i}^{C}\right) \text { for } i \in\{\ell, h\} .
$$

Alternatively, agent $i$ can first make the first-best investment $y^{*}$ and then deviate by not agreeing to $T^{*}$. Also then the payoffs are determined by bargaining and equal (8) and (9) where $y^{J}$ and $y_{i}^{C}$ are replaced by $y^{*}$. Agent $i$ must be strictly better off by choosing $y_{i}^{\omega}$ rather than $y^{*}$ in the investment stage and, consequently, deviation in

\footnotetext{
${ }^{8}$ We continue to apply Nash bargaining payoffs also in the repeated game. These payoffs can arise e.g. when each party can make a take-it-or-leave-it offer with probability $\frac{1}{2}$, as in Halac (2012) and (2015). See Miller and Watson (2013) for a richer analysis of bargaining in repeated games.
} 
investment dominates deviation in transfer.

The agents can find a suitable $T^{*}$ that satisfies both agents' ICs as long as the aggregate IC is satisfied. Summing up (6) and (7) we obtain

$$
S^{*} \geq(1-\delta)\left(P_{\ell}^{d, \omega}+P_{h}^{d, \omega}\right)+\delta S^{p, \omega}
$$

where $S^{*}$ is the first-best joint surplus and $S^{p, \omega}$ is the joint surplus in the punishment path. Equivalently, the first-best investments can be sustained in SPE if and only if

$$
\delta \geq \frac{G^{\omega}}{G^{\omega}+V^{\omega}} \equiv \underline{\delta}^{\omega}
$$

where $G^{\omega}=\left(P_{h}^{d, \omega}+P_{\ell}^{d, \omega}-S^{*}\right)$ is the aggregate gain from deviation and $V^{\omega}=$ $\left(S^{*}-S^{p, \omega}\right)$ is the value of the relationship (as compared to one governed by a spot contract). ${ }^{9}$ In the limit as $\delta \rightarrow 1$, the first-best investments are sustainable under any ownership structure. Our aim is to find an ownership structure which sustains the first-best investments for the lowest discount factor, that is, for which $\underline{\delta}^{\omega}$ is minimized.

It is useful to first examine the gain from deviation and the value of the relationship.

Lemma $1(i) G^{J}<G^{C}$,

(ii) $V^{C}<V^{J}$ if and only if $\gamma<1.5$.

There are two sources to the gain from deviation. The first part comes from the ability to expropriate from the other agent's first-best investment. Under joint ownership, according to (8), the agent can expropriate half of the joint value of the

\footnotetext{
${ }^{9}$ We adopt Halac's (2012) terminology but define the value of the relationship as the difference between trade governed by relational and spot contract. In Halac (2012) the comparison is to outside opportunities. While separation is an applicable punishment in business relationships, it is almost nonexisting in the stable communities that manage CPRs.
} 
other agent's first-best investment while under common ownership, according to (9), he can expropriate the full individual value. However, adding these up amounts to $\left(\theta_{\ell}+\theta_{h}\right) y^{*}$ under both ownership structures. Therefore the difference in the gain from deviation comes from the second source, the payoff earned from the agent's own second-best investment, as can be seen from the following equation (which is derived in (37) in the Appendix).

$$
G^{C}-G^{J}=\left[\theta_{h} y_{h}^{C}-c\left(y_{h}^{C}\right)\right]+\left[\theta_{\ell} y_{\ell}^{C}-c\left(y_{\ell}^{C}\right)\right]-2\left[\frac{1}{2}\left(\theta_{\ell}+\theta_{h}\right) y^{J}-c\left(y^{J}\right)\right]
$$

When $\theta_{\ell}=\theta_{h}$, the ownership structures are equivalent (see (3) and (5)) and $G^{C}=G^{J}$. Using the envelope theorem we can show that

$$
\frac{\partial\left(G^{C}-G^{J}\right)}{\partial \theta_{h}}=y_{h}^{C}-y^{J}>0
$$

and therefore $G^{C}>G^{J}$ for any $\theta_{\ell}<\theta_{h}$. The gain from deviation is higher under common ownership because agent $h$ gets his full valuation from his contribution to the public good while under joint ownership he has to share his high valuation in bargaining. There is the opposite effect for agent $\ell$ but it is of a smaller magnitude. ${ }^{10}$

Lemma $1(i i)$ is the mirror image of Proposition 1. Since the joint surplus is lower in the static game under joint ownership for $\gamma<1.5$, joint ownership maximizes the value of the relationship in this parameter range.

Proposition 2 derives the optimal ownership structure that minimizes the gain from deviation relative to the value of the relationship.

\footnotetext{
10 This is because agent $i$ 's payoff from his own investment is convex in the surplus share. Agent $i$ 's payoff from his own investment is $\sigma y_{i}(\sigma)-c\left(y_{i}(\sigma)\right)=\sigma(\sigma / \gamma)^{1 /(\gamma-1)}-(\sigma / \gamma)^{\gamma /(\gamma-1)}=(\gamma-$ 1) $(\sigma / \gamma)^{\gamma /(\gamma-1)}$ where $\sigma$ denotes the surplus share. It is straigtforward to verify that this payoff is convex in $\sigma$.
} 
Proposition 2 In the repeated game common ownership dominates joint ownership if and only if $\gamma>2$.

According to Lemma 1, joint ownership provides both the maximal value of the relationship and the minimal gain from deviation for $\gamma<1.5$. Then, unambiguously, $\underline{\delta}^{J}<\underline{\delta}^{C}$. For higher values of $\gamma$ there is a trade-off: joint ownership minimizes the gain from deviation but also minimizes the value of the relationship. Proposition 2 shows that joint ownership is optimal also for $1.5 \leq \gamma<2$. The result depends on the curvature of the marginal cost. If $\gamma<2$, the marginal cost is concave implying that the investment is elastic to the surplus share. ${ }^{11}$ Consequently, the difference between the first-best investment and the punishment investment is large and the value of the relationship is high under any ownership structure. Therefore, minimizing the gain from deviation provides the best incentives and joint ownership is optimal.

If $\gamma>2$, the marginal cost is convex and the investment is inelastic to the surplus share. Then the value of the relationship is low as the difference between the firstbest investment and the punishment investment is small. Ensuring that the relational contract is self-enforcing requires then an ownership structure that maximizes the value of the relationship. It furthermore depends on $\gamma$ which ownership structure does this. In this parameter range common ownership maximizes the value of the relationship and is therefore optimal.

Let us now compare the optimal ownership structure in the static and in the repeated game. In the static game joint ownership dominates when equalizing the agents' investments is cost-effective $(\gamma>1.5)$ while in the repeated game joint ownership is optimal if $\gamma<2$. Interestingly, the parameter ranges are partially overlapping and for $1.5<\gamma<2$ joint ownership is optimal both in the static and in the repeated game.

\footnotetext{
${ }^{11}$ Agent's optimal investment in the static game is $y=(\sigma / \gamma)^{1 /(\gamma-1)}$. The elasticity of investment to the surplus share is given by $(\partial y / \partial \sigma)(\sigma / y)=1 /(\gamma-1)$.
} 
Note that our model puts all the curvature in the cost function. It is straightforward to show that the model is isomorphic to a formulation where the value of the public good is $\left(\theta_{\ell}+\theta_{h}\right)\left[\left(y_{\ell}\right)^{\frac{1}{\gamma}}+\left(y_{h}\right)^{\frac{1}{\gamma}}\right]$ and the investment costs are linear, $c\left(y_{i}\right)=y_{i}{ }^{12}$ Then $\gamma>2$ implies that the marginal value of the investment is low. This is consistent with a stylized fact of common ownership: the value of the CPR cannot be increased much by investment (Netting 1976; Ostrom 1990, p. 63). The use of Alpine hillsides for communal grazing lands in Switzerland is an example of such common ownership. Similarly, in the main model $\gamma>2$ implies that the marginal cost of investment is high. In Section 7 we argue that this matches well with communally owned irrigation systems in Nepal and the Philippines. To combine both cases, we can say that common ownership is optimal when the marginal return to investment is low.

Our results also show that joint ownership is optimal when the marginal return to investment is high $(\gamma<2)$. Horizontal research joint venture (RJV) is a leading example of joint ownership of a public good. ${ }^{13}$ Marginal return to investment is high(er) consistent with our result. ${ }^{14}$

\section{Single ownership}

In the main model we have compared shared ownership structures. We now turn our attention to an ownership structure where either agent $\ell$ or $h$ is the single owner.

\footnotetext{
${ }^{12}$ In this alternative formulation, under common ownership we have $y_{i}^{C}=c\left(y_{i}^{C}\right)=\left(\frac{\theta_{i}}{\gamma}\right)^{\frac{\gamma}{\gamma-1}}$. Joint surplus equals $S^{C}=\left(\theta_{\ell}+\theta_{h}\right)\left[\left(\frac{\theta_{\ell}}{\gamma}\right)^{\frac{1}{\gamma-1}}+\left(\frac{\theta_{h}}{\gamma}\right)^{\frac{1}{\gamma-1}}\right]-\left(\frac{\theta_{\ell}}{\gamma}\right)^{\frac{\gamma}{\gamma-1}}-\left(\frac{\theta_{h}}{\gamma}\right)^{\frac{\gamma}{\gamma-1}}$ as in the main model. Furthermore, the value of the investment, $\left(y_{i}^{C}\right)^{\frac{1}{\gamma}}$, is also elastic to the surplus share if and only if $\gamma<2$

${ }^{13}$ In a horizontal RJV innovation becomes a public good for the partners. By contrast, in a vertical RJV an inventor and a developer cooperate and, since only the developer can commercialize the innovation, a private goods model is appropriate as in Aghion and Tirole (1994).

${ }^{14}$ See e.g. Hagedoorn (1996) and (2002) for evidence.
} 
Single ownership can be equivalent to either government ownership or privatization. ${ }^{15}$ In some applications it is appropriate to assume that the government has a higher valuation for the public good than a private party. In other applications the government may well have a lower valuation than e.g. an NGO.

We assume that if bargaining breaks down, proportion $\mu$ of the non-owner's investment remains in the project, where $\mu \in[0,1) .{ }^{16}$ Therefore agent $k$ 's disagreement payoff under ownership by $i$ is $\theta_{k}\left(y_{i}+\mu y_{j}\right)$, where $k, i, j \in\{\ell, h\}$ and $i \neq j .{ }^{17}$

The Nash bargaining payoffs under ownership by agent $i$ are

$$
\begin{aligned}
& u_{i}^{i}=\frac{1}{2}\left(\theta_{\ell}+\theta_{h}\right)\left(y_{i}+y_{j}\right)+\frac{1}{2}\left(\theta_{i}-\theta_{j}\right)\left(y_{i}+\mu y_{j}\right)-c\left(y_{i}\right), \\
& u_{j}^{i}=\frac{1}{2}\left(\theta_{\ell}+\theta_{h}\right)\left(y_{i}+y_{j}\right)+\frac{1}{2}\left(\theta_{j}-\theta_{i}\right)\left(y_{i}+\mu y_{j}\right)-c\left(y_{j}\right) .
\end{aligned}
$$

Investment incentives in the static game are

$$
\begin{gathered}
\theta_{i}=c^{\prime}\left(y_{i}^{i}\right), \\
\frac{1}{2}\left(\theta_{\ell}+\theta_{h}\right)+\frac{1}{2}\left(\theta_{j}-\theta_{i}\right) \mu=c^{\prime}\left(y_{j}^{i}\right) .
\end{gathered}
$$

Note that common ownership is formally equivalent to single ownership when $\mu=1$.

\footnotetext{
${ }^{15}$ Note that here privatization means private ownership of a public good. Privatization can also mean transforming a public good to a private good. This type of privatization will be discussed in Section 6 .

${ }^{16}$ Now, unlike in the main model, it matters whether the investment is in physical or human capital. Investment in physical capital remains in the project if the investing agent leaves and $\mu=1$. Investment in human capital is embedded in the agent and, therefore, if the agent leaves, so does the human capital unless some of it has spilled over to the project. Therefore $\mu \in[0,1)$. Project-specific human capital includes e.g. engineering skills that are specific to the public good and relationships in the community.

${ }^{17}$ In the public goods context Nash bargaining has a credibility issue regarding ex post disagreement payoffs that we share with Besley and Ghatak (2001). If bargaining breaks down, the owner would benefit from giving access to the other agent so that also his investment contributes to the public good. The theory of contracts as reference points (Hart and Moore 2008; Hart 2009) provides a possible avenue to explain this ex post inefficiency arising due to the souring of the relationship.
} 
In both cases both agents' investments fully contribute to the value of the project even under disagreement - either because the access of common owners cannot be restricted or because the non-owner's investment is fully sunk in the project. We assume that $\mu \in[0,1)$ to make the analysis interesting.

It follows from (3), (5), (16) and (17) that

$$
\begin{aligned}
& y_{\ell}^{C}=y_{\ell}^{\ell}<y_{\ell}^{h} \leq y^{J}<y^{*}, \\
& y^{J} \leq y_{h}^{\ell}<y_{h}^{C}=y_{h}^{h}<y^{*} .
\end{aligned}
$$

(18) and (19) firstly replicate the main result of Besley and Ghatak (2001): $h$-ownership provides better incentives for both agents than $\ell$-ownership. Secondly, $h$-ownership dominates common ownership and therefore a repeated game is needed to provide a rationale for common ownership. Thirdly, $h$-ownership does not dominate joint ownership but both can be optimal in a static game. ${ }^{18}$

As previously, let us start by analyzing the gain from deviation and the value of the relationship.

Lemma 2 (i) $\max \left\{G^{J}, G^{h}\right\}<G^{C}<G^{\ell}$,

(ii) $\max \left\{V^{J}, V^{h}\right\}<V^{C}<V^{\ell}$ if and only if $\gamma>1.5$,

(iii) $V^{h}<V^{C}<\min \left\{V^{J}, V^{\ell}\right\}$ if and only if $\gamma<1.5$.

Lemma 2 shows that there are broadly two classes of ownership structures. First, the ownership structures that can be optimal in the static game - joint ownership and $h$-ownership - minimize the gain from deviation. Second, the ownership structures that are dominated in the static game - common ownership and $\ell$-ownership - maximize the value of the relationship for large $\gamma$. In line with the previous results, we will

\footnotetext{
${ }^{18}$ It is easy to show that joint ownership is optimal in the static game if and only if $\gamma>1.5$ and $\mu \geq \widetilde{\mu}$, where $0<\widetilde{\mu}<1$, and $h$-ownership is optimal otherwise.
} 
show that common ownership or $\ell$-ownership is optimal if $\gamma>2$ while joint ownership or $h$-ownership is optimal if $\gamma<2$.

Proposition 3 derives the optimal ownership structure.

\section{Proposition 3 In the repeated game}

(i) joint ownership is optimal if $\gamma<2$ and $\mu \rightarrow 1$,

(ii) ownership by the high-valuation agent is optimal if $\gamma<2$ and $\mu \rightarrow 0$,

(iii) common ownership is optimal if $\gamma>2$ and $\frac{\theta_{\ell}}{\theta_{h}} \rightarrow 0$ and

(iv) ownership by the low-valuation agent is optimal if $\gamma>2, \frac{\theta_{\ell}}{\theta_{h}} \rightarrow 1$ and $\mu \rightarrow 0$.

Proposition 3 shows that also single ownership can emerge as optimal in the repeated game. In the parameter range where the gain effect is important $(\gamma<2)$, $h$ ownership is optimal for small $\mu$ because it minimizes the gain from deviation. Agent $h$ 's deviation payoff under $h$-ownership is

$$
P_{h}^{d, h}=\frac{1}{2}\left(\theta_{h}+\theta_{\ell}\right)\left(y_{h}^{h}+y^{*}\right)+\frac{1}{2}\left(\theta_{h}-\theta_{\ell}\right)\left(y_{h}^{h}+\mu y^{*}\right)-c\left(y_{h}^{h}\right)
$$

When agent $\ell$ 's first-best investment is largely embedded in himself $(\mu \rightarrow 0), h$ 's ability to extract from it is limited. ${ }^{19}$ While for high values of $\mu$ the gain from deviation under joint ownership (which does not depend on $\mu$ ) is lower than the gain from deviation under $h$-ownership (which is increasing in $\mu$ ) and joint ownership is optimal.

Ownership by the low-valuation agent can be optimal for $\gamma>2$ but only if $\theta_{\ell}$ is not too low compared to $\theta_{h}$. Here the effects are more subtle as the optimal ownership structure does not simply maximize the value of the relationship $\left(V^{\ell}>V^{C}\right.$ and $G^{\ell}>$ $G^{C}$ for all parameter values). The difference between agent $\ell^{\prime}$ s deviation payoffs,

\footnotetext{
${ }^{19}$ Note that for agent $\ell$ there is the opposite effect, $\partial P_{\ell}^{d, h} / \partial \mu<0$, but it is of a smaller magnitude so that $\partial G^{h} / \partial \mu>0$ as verified by (47).
} 
taking into account that $y_{\ell}^{\ell}=y_{\ell}^{C}$, is equal to

$$
P_{\ell}^{d, \ell}-P_{\ell}^{d, C}=\frac{1}{2}\left(\theta_{h}-\theta_{\ell}\right)(1-\mu) y^{*}>0
$$

Agent $\ell$ can extract more from $h$ 's first-best investment under $\ell$-ownership since in bargaining $\theta_{h} y^{*}$ is shared while under common ownership $\ell$ just gets his own low valuation, $\theta_{\ell} y^{*}$. For a similar reason $\ell$ 's deviation payoff is more responsive to a change in $\theta_{\ell}$ under common ownership, $\partial\left(P_{\ell}^{d, \ell}-P_{\ell}^{d, C}\right) / \partial \theta_{\ell}<0$, and therefore higher $\theta_{\ell}$ increases $\ell$ 's deviation payoff - and the aggregate gain from deviation - more under common ownership favouring $\ell$-ownership. That is why $\ell$-ownership is optimal for $\frac{\theta_{\ell}}{\theta_{h}} \rightarrow 1$. While common ownership emerges as optimal when the (marginal) effect on the value of the relationship is dominant. The difference between agent $\ell$ 's punishment payoffs is equal to

$$
P_{\ell}^{p, \ell}-P_{\ell}^{p, C}=\frac{1}{2}\left[\left(\theta_{h}+\theta_{\ell}\right)+\left(\theta_{\ell}-\theta_{h}\right) \mu\right] y_{h}^{\ell}-\theta_{\ell} y_{h}^{C}
$$

As above, $\partial\left(P_{\ell}^{p, \ell}-P_{\ell}^{p, C}\right) / \partial \theta_{\ell}<0$ since the change in $\theta_{\ell}$ is shared in bargaining under $\ell$-ownership while it has the full effect on $\ell$ 's payoff under common ownership. Therefore lower $\theta_{\ell}$ decreases $\ell$ 's punishment payoff more under common ownership than under $\ell$-ownership. ${ }^{20}$ Therefore the value of the relationship increases more under common ownership when $\frac{\theta_{\ell}}{\theta_{h}} \rightarrow 0$ and common ownership is optimal.

Figure 1 presents simulation results which show that the results of Proposition 3 hold also for intermediate values of $\mu$ and $\frac{\theta_{\ell}}{\theta_{h}} \cdot{ }^{21}$

\footnotetext{
${ }^{20}$ Note also that $\partial^{2}\left(P_{\ell}^{p, \ell}-P_{\ell}^{p, C}\right) / \partial\left(\theta_{\ell}\right)^{2}>0$ and $\partial^{2}\left(P_{\ell}^{d, \ell}-P_{\ell}^{d, C}\right) / \partial\left(\theta_{\ell}\right)^{2}<0$ which explains why the marginal effect on the value of the relationship is dominant for low $\theta_{\ell}$ and the marginal gain effect is dominant for high $\theta_{\ell}$.

${ }^{21}$ Figure $1(\mathrm{a})$ is drawn for small $\frac{\theta_{\ell}}{\theta_{h}}$ so that common ownership is optimal for all $\mu$ if $\gamma>2$. Figure 1 (b) is drawn for small $\mu$ so that $h$-ownership is optimal for all $\frac{\theta_{\ell}}{\theta_{h}}$ if $\gamma<2$. Simulation results also show that $\mu \rightarrow 0$ is not a necessary condition for $\ell$-ownership to be optimal (Proposition $5(i v)$ ).
} 


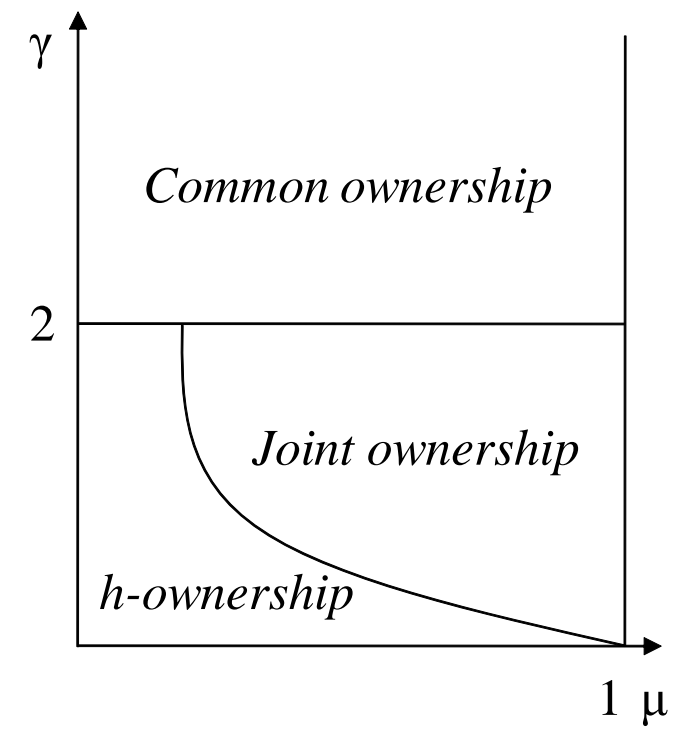

Figure 1(a)

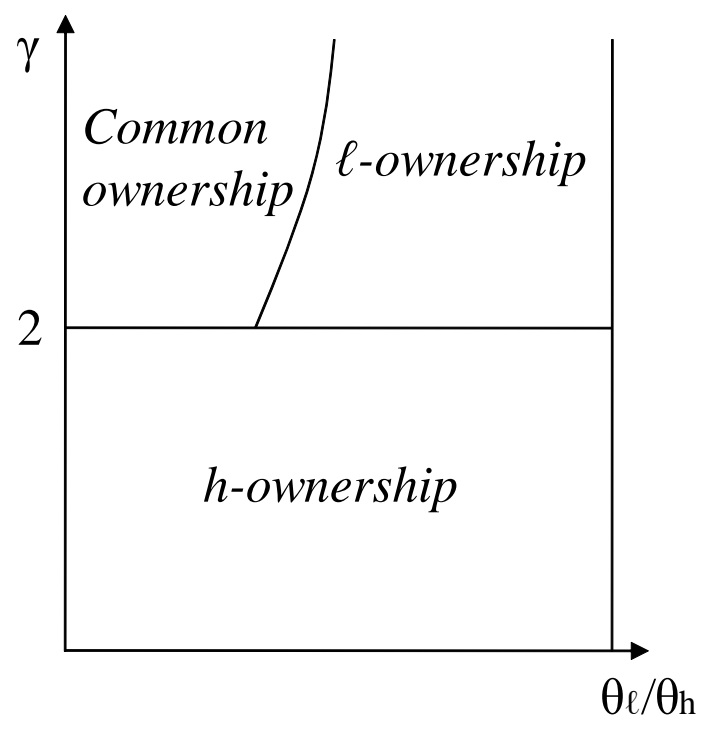

Figure 1(b)

Even when we include single ownership in the analysis, common ownership continues to be optimal in the relevant case of low marginal return to investments - as long as the agents' valuations of the public good are not too homogeneous. This additional condition requires further discussion. In the empirical literature there is no consensus on the role of heterogeneity in the management of CPRs (see e.g. Poteete and Ostrom 2004; Ruttan 2006, 2008). Different types of heterogeneity can have different effects. For example, economic inequality may have a positive effect ${ }^{22}$ while sociocultural heterogeneity may have a negative effect. In our model the agents differ only in the valuation of the public good. The valuation difference can arise from economic inequality (e.g. the value of irrigation system depends on the agent's land

\footnotetext{
${ }^{22}$ See also Baland and Platteau (2003) for theoretical analysis where the effect of income inequality depends on the model setup.
} 
endowment) and therefore our result is consistent with the empirical results.

Joint ownership also remains optimal for high marginal return to investments. The additional requirement is that the investments are largely sunk in the project. This matches well with R\&D since innovation can typically be commercialized without the presence of the other party of the horizontal RJV.

\section{Renegotiation}

In the main model we have applied trigger strategies and assumed that there is no renegotiation in the punishment path. We now introduce renegotiation and allow the deviator to pay a monetary transfer to the other agent to restore cooperation. ${ }^{23}$ This is a natural way to model renegotiation as in common property arrangements offenders typically pay a fine to the community. Ostrom (1990, p. 90) identifies graduated sanctions as one of the design principles of successful CPRs. Our analysis focuses on the maximal fine.

The agents' strategies are the following.

Phase 1: Invest $y^{*}$ and pay (or receive) $T^{*}$. If agent $i$ deviates, start Phase 2.

Phase 2:

Agent $j \neq i$ : If agent $i$ pays fine $F^{i, \omega}$ at the beginning of the period, go back to Phase 1. Otherwise, invest $y_{j}^{p, \omega}$ and start Phase 2 in the next period.

Agent $i$ : Pay $F^{i, \omega}$ to agent $j$ and go back to Phase 1 .

If any player deviates in Phase 2, re-start Phase 2 against that player.

After deviating in investment, agent $i$ can restore cooperation by paying fine $F^{i, \omega}$ to agent $j$ at the beginning of the following period. If agent $i$ does not pay $F^{i, \omega}$, then agent $j$ will choose punishment investment $y_{j}^{p, \omega}$ in that period. We examine two

\footnotetext{
${ }^{23}$ Our approach draws from the private goods case of Blonski and Spagnolo (2007).
} 
alternatives for the punishment investment. Mild punishment implies $y_{j}^{p, \omega}=y_{j}^{\omega}$ while severe punishment investment can be as low as zero. We argue that mild punishment is a reasonable assumption for common property arrangements as the community typically depends on the CPR for a major portion of their livelihood (Ostrom 2002) and maintenance is critical, especially given the relatively poor quality materials used in many CPRs. For example, in the case of the long-standing irrigation systems in the Philippines (Zanjera irrigation communities), dams are constructed of bamboo poles, banana leaves, sand and rock, and as a result require continual maintenance (Ostrom 1990, p. 83). Mild punishment may not be equally reasonable in other contexts, and we therefore examine also severe punishment. In $R \& D$ cooperation zero punishment investment would imply delaying the project.

\section{$5.1 \quad$ Mild punishment}

Suppose agent $h$ has deviated in investment. The IC for paying the fine $F^{h, \omega}$ is

$-(1-\delta) F^{h, \omega}+\left[2 \theta_{h} y^{*}-c\left(y^{*}\right)-T^{*}\right] \geq(1-\delta) P_{h}^{p, \omega}+\delta\left\{-(1-\delta) F^{h, \omega}+\left[2 \theta_{h} y^{*}-c\left(y^{*}\right)-T^{*}\right]\right\}$

If agent $h$ pays $F^{h, \omega}$, cooperation is restored immediately. Otherwise, agent $h$, bestresponding to $y_{\ell}^{\omega}$, earns his static payoff $P_{h}^{p, \omega}$ and, using the one-shot deviation principle, the restoration of cooperation is postponed by one period. From (21) we can solve for the maximal fine that agent $h$ would be willing to pay.

$$
F^{h, \omega}=\frac{\left[2 \theta_{h} y^{*}-c\left(y^{*}\right)-T^{*}\right]-P_{h}^{p, \omega}}{(1-\delta)}
$$


In a similar manner we can derive $F^{\ell, \omega}$.

$$
F^{\ell, \omega}=\frac{\left[2 \theta_{\ell} y^{*}-c\left(y^{*}\right)+T^{*}\right]-P_{\ell}^{p, \omega}}{(1-\delta)}
$$

The ICs for investing $y^{*}$ are now

$$
\begin{gathered}
{\left[2 \theta_{h} y^{*}-c\left(y^{*}\right)-T^{*}\right] \geq(1-\delta) P_{h}^{d, \omega}+\delta\left\{-(1-\delta) F^{h, \omega}+\left[2 \theta_{h} y^{*}-c\left(y^{*}\right)-T^{*}\right]\right\},} \\
{\left[2 \theta_{\ell} y^{*}-c\left(y^{*}\right)+T^{*}\right] \geq(1-\delta) P_{\ell}^{d, \omega}+\delta\left\{-(1-\delta) F^{\ell, \omega}+\left[2 \theta_{\ell} y^{*}-c\left(y^{*}\right)+T^{*}\right]\right\} .}
\end{gathered}
$$

Summing up (24) and (25), we obtain the aggregate IC.

$$
\delta\left(F^{h, \omega}+F^{\ell, \omega}\right) \geq P_{h}^{d, \omega}+P_{\ell}^{d, \omega}-S^{*}
$$

Finally, substituting (22) and (23) in (26) we obtain

$$
\delta \geq \frac{P_{h}^{d, \omega}+P_{\ell}^{d, \omega}-S^{*}}{P_{h}^{d, \omega}+P_{\ell}^{d, \omega}-S^{p, \omega}}=\frac{G^{\omega}}{G^{\omega}+V^{\omega}} .
$$

(27) is equivalent to (11) and therefore our previous results are robust to introducing renegotiation. Since all the surplus from renegotiation goes to the non-defecting party, the deviator's continuation payoff is equal to his continuation payoff without renegotiation, that is, his payoff with trigger strategies. Note also that renegotiation of ownership structure after deviation is not optimal since the fine restores ex post efficiency. 


\subsection{Severe punishment}

In Appendix A.2 we allow for zero punishment investment and examine when it is incentive compatible. We show that the critical discount factor changes to

$$
\delta \geq \frac{P_{h}^{d, \omega}+P_{\ell}^{d, \omega}-S^{*}}{P_{h}^{d, \omega}+P_{\ell}^{d, \omega}-P_{h}^{p, \omega}\left(y_{h}^{\omega}, 0\right)-P_{\ell}^{p, \omega}\left(y_{\ell}^{\omega}, 0\right)}=\frac{G^{\omega}}{\left(\theta_{\ell}+\theta_{h}\right) y^{*}}
$$

With severe punishment the optimal ownership structure minimizes the gain from deviation. Therefore joint ownership dominates common ownership. We argue that severe punishment is not reasonable for CPRs but may be appropriate in e.g. R\&D cooperation. The optimal ownership structure is then joint ownership if the investments are largely sunk in the project ( $\mu$ is high) or $h$-ownership if the investments are mainly in human capital that does not spill over to the project ( $\mu$ is low).

\section{Public vs. private goods}

In this section we compare the results of the repeated game for public and private goods, the latter analyzed in Halonen (2002). In the static game the results differ significantly. In the private goods case (Grossman and Hart 1986; Hart and Moore 1990; Hart 1995) it is optimal to concentrate ownership in the hands of the agent with an important investment. To the contrary, Besley and Ghatak (2001) show that it can be optimal for the high-valuation agent to own the public good even when only the low-valuation agent has an investment. Despite these differences, the private and public goods cases are aligned in the repeated game: in both cases the results depend on whether $\gamma$ is greater or smaller than 2 .

With private goods the worst ownership structure of the static game (joint ownership) is optimal for $\gamma>2$ because the value of the relationship is maximized. While 
the optimal ownership structure of the static game (single ownership) provides the best incentives when $\gamma<2$ because it minimizes the gain from deviation. With public goods the results no longer depend on the tradeoff between the best and the worst ownership structure of the static game. First, common ownership can be optimal for $\gamma>2$ even when $\ell$-ownership is the worst ownership structure in the static game. Second, joint ownership can minimize the gain from deviation even when it is not optimal in the static game. Furthermore, with private goods the results of the static game hold also in the repeated game for $\gamma<2-$ as the gain from deviation is minimized. With public goods also the optimal ownership structure of the static game depends on $\gamma$ and therefore - as discussed in Section 3 - there is overlap only for $1.5<\gamma<2$. Despite these differences, the critical value for $\gamma$ remains the same.

In Section 4 we analyzed single ownership of public goods which can take the form of privatization. However, private ownership of private goods is often the interesting case. For example, land can remain as a public good (under common or government ownership) or it can be parceled to be a private good. In Switzerland it is the more productive arable lands in the mountain valleys that are privately owned (Netting 1976). This is consistent with Halonen (2002) where single ownership is optimal for private goods when $\gamma<2$.

\section{Common pool resources}

Our model applies well to CPRs such as irrigation systems and common lands, which require maintenance investments. We have shown that common ownership is optimal when the marginal return to maintenance investments is low $(\gamma>2)$. The marginal return can be low either because the marginal cost is high - as in the main model 
where the costs equal $\left(y_{i}\right)^{\gamma}$ - or because the marginal value is low - as in the alternative formulation where the value of the $\operatorname{CPR}$ is $\left(\theta_{\ell}+\theta_{h}\right)\left[\left(y_{\ell}\right)^{\frac{1}{\gamma}}+\left(y_{h}\right)^{\frac{1}{\gamma}}\right]$.

Most of the Alpine pasture in Switzerland has been under common ownership for centuries while arable land in the mountain valleys is typically privately owned. ${ }^{24}$ Netting (1976) identifies stylized facts of Alpine land ownership (see also Ostrom 1990, p. 63). He observes that while the yield of privately owned arable land can be increased by "irrigation, manuring, erosion control, crop rotation, and careful horticulture" (Netting 1976, p. 143), the possibility of improving the commonly owned Alpine grazing lands is low due to altitude, limited growing season and thin soils. Maintenance of the grazing lands includes distributing manure, renewing avalanche-damaged corrals and clearing access paths and roads. This stylized fact of common ownership is consistent with the version of our model where the value of the CPR cannot be increased much by investment when $\gamma>2$. Extensive common lands have existed for centuries also in Japan (McKean 1982; Ostrom 1990, p. 65). Also there the maintenance investments, including the annual burning of the grasslands, have a limited impact on the yield. ${ }^{25}$

The marginal return to investment can be low also due to high marginal cost. Then common ownership can be optimal even when it is possible to increase the value of the CPR significantly by investment. This possibility is not reflected in the above stylized fact (drawn from common lands). However, it is consistent with the communally owned irrigation systems in Nepal and the Philippines. ${ }^{26}$ The irrigation systems are very valuable to the local communities as their livelihood depends on irrigation. Furthermore, their value can be increased significantly by investments.

\footnotetext{
${ }^{24}$ In Valais, Switzerland, 95\% of Alpine pasture has been under common ownership (Netting 1976). Also in Italy most of the Alpine pasture has been commonly owned (Casari 2007).

${ }^{25}$ Communal marshes in Nonatola, Italy, are another example where the marginal value of investments is low. The marshes required "an incredible amount of work to cultivate yearly crops" (Tagliapietra 2011, p. 32).

${ }^{26}$ For more details see e.g. Yoder (1994) for Nepal and Ostrom (1990, p. 82-88) for the Philippines.
} 
Investments, however, are very costly. For example, one of the Zanjera irrigation communities in the Philippines constructs and maintains a 100-meter-long dam that spans the unpredictable Bacarra-Vintar River (Coward and Siy 1983; Ostrom 1990, p. 83). The technology used to construct the dam is labor-intensive, involving hundreds of people. Building materials include bamboo poles, banana leaves, sand and rock. Furthermore, the dam might have to be rebuilt up to four times a year. ${ }^{27}$ Additionally, the canals require regular maintenance including removing vegetation, gravel and siltation. The average contribution of the community members in 1980 was 37 days of work per person and, additionally, they contributed building materials. ${ }^{28}$ We argue that in this case the marginal cost of maintenance is high. The marginal cost arises from the time and effort taken away from cultivating privately owned fields. It is reasonable to assume that this marginal cost is high and, furthermore, increases at an increasing rate when the significant contribution of work is further increased, that is, $\gamma>2$.

Maintenance is provided communally in all the above examples. Required contributions by community members are typically proportional to the benefits they receive from the resource (e.g. proportional to the land irrigated or to the number of animals summered in the communal grazing lands). These communities have been successful in mobilizing a significant amount of labor regularly. For example, in the above Zanjera irrigation community compliance rate was $94 \%$ (Ostrom 1990, p. 86). Nonattendance is punished by fines paid to the community (Netting 1972; McKean 1982; Ostrom 1990, p. 86) unless an acceptable excuse is provided. ${ }^{29}$ For example, in the

\footnotetext{
${ }^{27}$ The dam is designed to self-destruct when the water rises above a certain level, thus preventing the canals from flooding.

${ }^{28}$ Another example of costly maintenance comes from the Chhatis Mauja irrigation system in Nepal where the desilting of the main canal in 1988 took 13 days and on average 760 community members were working each day (Yoder 1994, p. 67).

${ }^{29}$ In our model we have assumed that maintenance investments are observable. This is a reasonable assumption as attendance in the days that the community gathers to maintain the CPR is indeed
} 
villages of Japan studied by McKean (1982) the only acceptable excuses were illness, family tragedy or the absence of able-bodied adults.

Our analysis differs from Ostrom (1990) who examined numerous case studies of successful and unsuccessful CPRs and identified design principles that characterize the successful ones. These design principles - such as well-defined boundaries, graduated sanctions and low-cost conflict-resolution mechanisms ${ }^{30}$ - are largely endogenous. Our interest, to the contrary, is to find characteristics of exogenous maintenance technology for which common ownership emerges as optimal ownership structure. We show that the often cited examples of successful CPRs - irrigation systems in Nepal and the Philippines and common lands in Switzerland and Japan - do not only share similar design principles but can also be characterized by the same exogenous factor: low marginal return to maintenance investments.

In addition to good maintenance, successful CPRs also have to solve the potential overappropriation problem: overgrazing of common lands and overappropriation of water at the head of the irrigation system. ${ }^{31}$ Much of the previous research has focused on appropriation, e.g. Benhabib and Radner (1992) and Copeland and Taylor (2009). We complement this literature by introducing maintenance investments and examining when common ownership is an optimal ownership structure. ${ }^{32}$ While our public good model is "applicable to the provision of ... CPRs" (Ostrom 1990, p. 32), it cannot be used to analyze appropriation.

observable. Furthermore, the work is often organized so that the work teams can monitor the progress of other teams who have been given a task of a similar size (Ostrom 1990, p. 85).

${ }^{30}$ Other design principles are congruence between appropriation and provision rules and local conditions, collective-choice arrangements, monitoring, minimal recognition of rights to organize and nested enterprises (Ostrom 1990, p. 90).

${ }^{31}$ Some CPRs, such as fisheries, do not require any significant maintenance.

${ }^{32}$ Ostrom and Gardner (1993) examine the interaction of maintenance and appropriation in a communally owned irrigation system. They find that costly maintenance is helpful for reaching equitable access to irrigation. 


\section{Conclusions}

In this paper we have analyzed ownership of public goods, in particular common ownership. We show that common ownership cannot be optimal in the static game. However, consistent with the literature which has used insights from repeated games to understand successful management of CPRs (Ostrom 1990; Ostrom, Gardner and Walker 1994), we show that a repeated game can provide a rationale for common ownership. We find that common ownership is optimal when the marginal cost of investment is convex, that is when the investment is inelastic to the surplus share. Consequently, the value of the relationship is low as the one-shot investment is not significantly lower than the first-best investment. Common ownership is then optimal as it maximizes the value of the relationship.

In our model, convexity of marginal cost implies that the marginal return to investment is low. This result is consistent with an important stylized fact of common ownership, that the value of the CPR cannot be increased much by investment, as in the Alpine grazing lands in Switzerland (Netting 1976; Ostrom 1990, p. 63). Our result furthermore complements the existing literature by showing that common ownership can be optimal even when it is possible to increase the value of the CPR significantly by investment if the marginal cost of the investment is high, as in the irrigation systems in Nepal and the Philippines. Our analysis shows that these successful CPRs share the same exogenous characteristic - low marginal return to maintenance investments - in addition to similar design principles identified by Ostrom (1990).

Our analysis of public goods applies to provision and maintenance of CPRs. An important direction for future work is to extend the analysis to CPRs which are similar to public goods in the difficulty of excluding beneficiaries but differ in the possibility

of overuse. Also, future work could explore the difference between local and global 
CPRs - resources that go beyond national jurisdictions (e.g. earth's oceans and global climate). ${ }^{33}$ Global CPRs not only differ from local CPRs in terms of characteristics but also pose different challenges. ${ }^{34}$

\footnotetext{
${ }^{33}$ Ostrom's design principles were largely informed by research in local CPRs.

${ }^{34}$ See Stern (2011) for a useful discussion of the differences between global and local CPRs and the applicability of Ostrom's design principles to global CPRs.
} 


\section{A Appendix}

\section{A.1 Proofs}

Firstly, the explicit forms of the investments derived in (1), (3) and (5) are

$$
\begin{gathered}
y^{*}=\left(\frac{\theta_{\ell}+\theta_{h}}{\gamma}\right)^{\frac{1}{\gamma-1}}, \\
y^{J}=\left(\frac{\theta_{\ell}+\theta_{h}}{2 \gamma}\right)^{\frac{1}{\gamma-1}}, \\
y_{\ell}^{C}=\left(\frac{\theta_{\ell}}{\gamma}\right)^{\frac{1}{\gamma-1}}, \\
y_{h}^{C}=\left(\frac{\theta_{h}}{\gamma}\right)^{\frac{1}{\gamma-1}} .
\end{gathered}
$$

\section{Proof of Proposition 1.}

Denote by $S^{\omega}$ joint surplus under ownership structure $\omega \in\{J, C\} . S^{J}>S^{C}$ if and only if

$$
2\left(\theta_{\ell}+\theta_{h}\right) y^{J}-2 c\left(y^{J}\right)>\left(\theta_{\ell}+\theta_{h}\right)\left(y_{\ell}^{C}+y_{h}^{C}\right)-c\left(y_{\ell}^{C}\right)-c\left(y_{h}^{C}\right)
$$

Substituting $\theta_{h}=\alpha \theta_{\ell}$, where $\alpha>1$, and (30) - (32) in (33) we obtain

$$
2(\alpha+1) \theta_{\ell}\left(\frac{(\alpha+1) \theta_{\ell}}{2 \gamma}\right)^{\frac{1}{\gamma-1}}-2\left(\frac{(\alpha+1) \theta_{\ell}}{2 \gamma}\right)^{\frac{\gamma}{\gamma-1}}>
$$




$$
(\alpha+1) \theta_{\ell}\left[\left(\frac{\alpha \theta_{\ell}}{\gamma}\right)^{\frac{1}{\gamma-1}}+\left(\frac{\theta_{\ell}}{\gamma}\right)^{\frac{1}{\gamma-1}}\right]-\left(\frac{\alpha \theta_{\ell}}{\gamma}\right)^{\frac{\gamma}{\gamma-1}}-\left(\frac{\theta_{\ell}}{\gamma}\right)^{\frac{\gamma}{\gamma-1}}
$$

which is equivalent to

$$
\begin{gathered}
2(\alpha+1)\left(\frac{\alpha+1}{2 \gamma}\right)^{\frac{1}{\gamma-1}}-2\left(\frac{\alpha+1}{2 \gamma}\right)^{\frac{\gamma}{\gamma-1}}> \\
(\alpha+1)\left[\left(\frac{\alpha}{\gamma}\right)^{\frac{1}{\gamma-1}}+\left(\frac{1}{\gamma}\right)^{\frac{1}{\gamma-1}}\right]-\left(\frac{\alpha}{\gamma}\right)^{\frac{\gamma}{\gamma-1}}-\left(\frac{1}{\gamma}\right)^{\frac{\gamma}{\gamma-1}} .
\end{gathered}
$$

Multiplying by $\gamma^{\frac{1}{\gamma-1}}$ and rearranging, (34) is equivalent to $\psi_{s}(\alpha, \gamma)>\chi_{s}(\alpha, \gamma)$ where

$$
\begin{gathered}
\psi_{s}(\alpha, \gamma)=\frac{2 \gamma-1}{\gamma}\left(\frac{1}{2}\right)^{\frac{1}{\gamma-1}}(\alpha+1)^{\frac{\gamma}{\gamma-1}}-(\alpha+1) \alpha^{\frac{1}{\gamma-1}}+\frac{1}{\gamma} \alpha^{\frac{\gamma}{\gamma-1}} \\
\chi_{s}(\alpha, \gamma)=(\alpha+1)-\frac{1}{\gamma} .
\end{gathered}
$$

Note that $\psi_{s}(1, \gamma)=\chi_{s}(1, \gamma)=\frac{2 \gamma-1}{\gamma}$ and $\left.\frac{\partial \psi_{s}(\alpha, \gamma)}{\partial \alpha}\right|_{\alpha=1}=\left.\frac{\partial \chi_{s}(\alpha, \gamma)}{\partial \alpha}\right|_{\alpha=1}=1$ given

$$
\frac{\partial \psi_{s}(\alpha, \gamma)}{\partial \alpha}=\frac{2 \gamma-1}{\gamma-1}\left(\frac{1}{2}\right)^{\frac{1}{\gamma-1}}(\alpha+1)^{\frac{1}{\gamma-1}}-\alpha^{\frac{1}{\gamma-1}}-\frac{1}{\gamma-1} \alpha^{\frac{2-\gamma}{\gamma-1}}
$$

In the Online Appendix we establish that $\partial^{2} \psi_{s}(\alpha, \gamma) / \partial \alpha^{2}>0$ for any $\alpha>1$ if and only if $\gamma>1.5$. Since $\psi_{s}(\alpha, \gamma)$ and $\chi_{s}(\alpha, \gamma)$ are tangent at $\alpha=1$ and $\chi_{s}(\alpha, \gamma)$ is linear and increasing in $\alpha, \psi_{s}(\alpha, \gamma)>\chi_{s}(\alpha, \gamma)$ for any $\alpha>1$ if and only if $\gamma>1.5$. Consequently, $S^{J}>S^{C}$ if and only if $\gamma>1.5$. Q.E.D.

\section{Proof of Lemma 1.}

$(i)$

$$
\begin{aligned}
G^{C} & =\left[\theta_{h}\left(y^{*}+y_{h}^{C}\right)-c\left(y_{h}^{C}\right)\right]+\left[\theta_{\ell}\left(y^{*}+y_{\ell}^{C}\right)-c\left(y_{\ell}^{C}\right)\right]-\left[2\left(\theta_{\ell}+\theta_{h}\right) y^{*}-2 c\left(y^{*}\right)\right] \\
& =\left[\theta_{h} y_{h}^{C}-c\left(y_{h}^{C}\right)\right]-\left[\theta_{h} y^{*}-c\left(y^{*}\right)\right]+\left[\theta_{\ell} y_{\ell}^{C}-c\left(y_{\ell}^{C}\right)\right]-\left[\theta_{\ell} y^{*}-c\left(y^{*}\right)\right]>0 .
\end{aligned}
$$


$G^{C}>0$ since $y_{h}^{C}$ maximizes the first term in square brackets and $y_{\ell}^{C}$ maximizes the third term. Similarly, we can derive $G^{J}$.

$$
G^{J}=2\left[\frac{1}{2}\left(\theta_{\ell}+\theta_{h}\right) y^{J}-c\left(y^{J}\right)\right]-2\left[\frac{1}{2}\left(\theta_{\ell}+\theta_{h}\right) y^{*}-c\left(y^{*}\right)\right]>0
$$

Therefore

$$
G^{C}-G^{J}=\left[\theta_{h} y_{h}^{C}-c\left(y_{h}^{C}\right)\right]+\left[\theta_{\ell} y_{\ell}^{C}-c\left(y_{\ell}^{C}\right)\right]-2\left[\frac{1}{2}\left(\theta_{\ell}+\theta_{h}\right) y^{J}-c\left(y^{J}\right)\right]
$$

(13) in Section 3 proves that $G^{C}>G^{J}$ for any $\theta_{h}>\theta_{\ell}$.

(ii) According to Proposition $1 S^{p, J}>S^{p, C}$ if and only if $\gamma>1.5$ and, consequently, $V^{J}<V^{C}$ if and only if $\gamma>1.5$. Q.E.D.

\section{Proof of Proposition 2.}

To derive the explicit form of $\underline{\delta}^{J}$ we first substitute (29) and (30) in (36) obtaining

$$
\begin{aligned}
G^{J}= & 2\left[\frac{1}{2}\left(\theta_{\ell}+\theta_{h}\right)\left(\frac{\theta_{\ell}+\theta_{h}}{2 \gamma}\right)^{\frac{1}{\gamma-1}}-\left(\frac{\theta_{\ell}+\theta_{h}}{2 \gamma}\right)^{\frac{\gamma}{\gamma-1}}\right] \\
& -2\left[\frac{1}{2}\left(\theta_{\ell}+\theta_{h}\right)\left(\frac{\theta_{\ell}+\theta_{h}}{\gamma}\right)^{\frac{1}{\gamma-1}}-\left(\frac{\theta_{\ell}+\theta_{h}}{\gamma}\right)^{\frac{\gamma}{\gamma-1}}\right] \\
= & \left(\frac{\theta_{\ell}+\theta_{h}}{\gamma}\right)^{\frac{\gamma}{\gamma-1}}\left[(\gamma-1)\left(\frac{1}{2}\right)^{\frac{1}{\gamma-1}}-(\gamma-2)\right]>0 .
\end{aligned}
$$

(38) is positive by (36). 


$$
\begin{aligned}
G^{J}+V^{J} \text { equals } & \\
G^{J}+V^{J}= & 2\left[\frac{1}{2}\left(\theta_{\ell}+\theta_{h}\right) y^{J}-c\left(y^{J}\right)\right]-2\left[\frac{1}{2}\left(\theta_{\ell}+\theta_{h}\right) y^{*}-c\left(y^{*}\right)\right] \\
& +2\left[\left(\theta_{\ell}+\theta_{h}\right) y^{*}-c\left(y^{*}\right)\right]-2\left[\left(\theta_{\ell}+\theta_{h}\right) y^{J}-c\left(y^{J}\right)\right] \\
= & \left(\theta_{\ell}+\theta_{h}\right)\left(y^{*}-y^{J}\right) \\
= & \left(\frac{1}{\gamma}\right)^{\frac{1}{\gamma-1}}\left(\theta_{\ell}+\theta_{h}\right)^{\frac{\gamma}{\gamma-1}}\left[1-\left(\frac{1}{2}\right)^{\frac{1}{\gamma-1}}\right] .
\end{aligned}
$$

Using (38) and (39) we obtain

$$
\underline{\delta}^{J}=\frac{G^{J}}{G^{J}+V^{J}}=\frac{(\gamma-1)\left(\frac{1}{2}\right)^{\frac{1}{\gamma-1}}-(\gamma-2)}{\gamma\left[1-\left(\frac{1}{2}\right)^{\frac{1}{\gamma-1}}\right]} .
$$

Similarly, $G^{C}$ and $G^{C}+V^{C}$ equal

$$
\begin{aligned}
G^{C}= & {\left[\theta_{h}\left(\frac{\theta_{h}}{\gamma}\right)^{\frac{1}{\gamma-1}}-\left(\frac{\theta_{h}}{\gamma}\right)^{\frac{\gamma}{\gamma-1}}\right]+\left[\theta_{\ell}\left(\frac{\theta_{\ell}}{\gamma}\right)^{\frac{1}{\gamma-1}}-\left(\frac{\theta_{\ell}}{\gamma}\right)^{\frac{\gamma}{\gamma-1}}\right] } \\
& -\left[\left(\theta_{\ell}+\theta_{h}\right)\left(\frac{\theta_{\ell}+\theta_{h}}{\gamma}\right)^{\frac{1}{\gamma-1}}-2\left(\frac{\theta_{\ell}+\theta_{h}}{\gamma}\right)^{\frac{\gamma}{\gamma-1}}\right] \\
= & \left(\frac{\theta_{\ell}}{\gamma}\right)^{\frac{\gamma}{\gamma-1}}\left[(\gamma-1)+(\gamma-1) \alpha^{\frac{\gamma}{\gamma-1}}-(\gamma-2)(\alpha+1)^{\frac{\gamma}{\gamma-1}}\right],
\end{aligned}
$$

$$
\begin{aligned}
G^{C}+V^{C}= & {\left[\theta_{h} y_{h}^{C}-c\left(y_{h}^{C}\right)\right]+\left[\theta_{\ell} y_{\ell}^{C}-c\left(y_{\ell}^{C}\right)\right]-\left[\left(\theta_{\ell}+\theta_{h}\right) y^{*}-2 c\left(y^{*}\right)\right] } \\
& +\left[\left(\theta_{\ell}+\theta_{h}\right) 2 y^{*}-2 c\left(y^{*}\right)\right]-\left[\theta_{h}\left(y_{h}^{C}+y_{\ell}^{C}\right)-c\left(y_{h}^{C}\right)\right]-\left[\theta_{\ell}\left(y_{h}^{C}+y_{\ell}^{C}\right)-c\left(y_{\ell}^{C}\right)\right] \\
= & \theta_{h}\left(y^{*}-y_{\ell}^{C}\right)+\theta_{\ell}\left(y^{*}-y_{h}^{C}\right) \\
= & \left(\theta_{\ell}\right)^{\frac{\gamma}{\gamma-1}}\left(\frac{1}{\gamma}\right)^{\frac{1}{\gamma-1}}\left[\left((\alpha+1)^{\frac{1}{\gamma-1}}-\alpha^{\frac{1}{\gamma-1}}\right)+\alpha\left((\alpha+1)^{\frac{1}{\gamma-1}}-1\right)\right] .
\end{aligned}
$$


We have substituted in $\theta_{h}=\alpha \theta_{\ell}$. Using (41) and (42) we obtain

$$
\underline{\delta}^{C}=\frac{(\gamma-1)+(\gamma-1) \alpha^{\frac{\gamma}{\gamma-1}}-(\gamma-2)(\alpha+1)^{\frac{\gamma}{\gamma-1}}}{\gamma\left[\left((\alpha+1)^{\frac{1}{\gamma-1}}-\alpha^{\frac{1}{\gamma-1}}\right)+\alpha\left((\alpha+1)^{\frac{1}{\gamma-1}}-1\right)\right]} .
$$

In the Online Appendix we establish that $\underline{\delta}^{J}>\underline{\delta}^{C} \Leftrightarrow \chi_{r}(\alpha, \gamma)<\psi_{r}(\alpha, \gamma)$ where

$$
\begin{gathered}
\chi_{r}(\alpha, \gamma)=(\gamma-1)\left[1-\left(\frac{1}{2}\right)^{\frac{1}{\gamma-1}}\right]+\alpha\left[(\gamma-1)\left(\frac{1}{2}\right)^{\frac{1}{\gamma-1}}-(\gamma-2)\right], \\
\psi_{r}(\alpha, \gamma)=\left(\frac{1}{2}\right)^{\frac{1}{\gamma-1}}(\alpha+1)^{\frac{\gamma}{\gamma-1}}-(\gamma-1) \alpha^{\frac{\gamma}{\gamma-1}}\left[1-\left(\frac{1}{2}\right)^{\frac{1}{\gamma-1}}\right] \\
-\alpha^{\frac{1}{\gamma-1}}\left[(\gamma-1)\left(\frac{1}{2}\right)^{\frac{1}{\gamma-1}}-(\gamma-2)\right] .
\end{gathered}
$$

Differentiating with respect to $\alpha$ gives

$$
\begin{gathered}
\frac{\partial \chi_{r}(\alpha, \gamma)}{\partial \alpha}=\left[(\gamma-1)\left(\frac{1}{2}\right)^{\frac{1}{\gamma-1}}-(\gamma-2)\right]>0 \\
\frac{\partial \psi_{r}(\alpha, \gamma)}{\partial \alpha}=\frac{\gamma}{\gamma-1}\left(\frac{1}{2}\right)^{\frac{1}{\gamma-1}}(\alpha+1)^{\frac{1}{\gamma-1}}-\gamma \alpha^{\frac{1}{\gamma-1}}\left[1-\left(\frac{1}{2}\right)^{\frac{1}{\gamma-1}}\right] \\
-\frac{1}{\gamma-1} \alpha^{\frac{2-\gamma}{\gamma-1}}\left[(\gamma-1)\left(\frac{1}{2}\right)^{\frac{1}{\gamma-1}}-(\gamma-2)\right]
\end{gathered}
$$

Note that $\chi_{r}(1, \gamma)=\psi_{r}(1, \gamma)=1$ and $\left.\frac{\partial \chi_{r}(\alpha, \gamma)}{\partial \alpha}\right|_{\alpha=1}=\left.\frac{\partial \psi_{r}(\alpha, \gamma)}{\partial \alpha}\right|_{\alpha=1}=\left[(\gamma-1)\left(\frac{1}{2}\right)^{\frac{1}{\gamma-1}}+(2-\gamma)\right]$.

Note also that (44) is positive by (38). In the Online Appendix we establish that $\partial^{2} \psi_{r}(\alpha, \gamma) / \partial \alpha^{2}>0$ for any $\alpha>1$ if and only if $\gamma>2$. Since $\chi_{r}(\alpha, \gamma)$ and $\psi_{r}(\alpha, \gamma)$ are tangent at $\alpha=1$ and $\chi_{r}(\alpha, \gamma)$ is linear and increasing in $\alpha, \psi_{r}(\alpha, \gamma)>\chi_{r}(\alpha, \gamma)$ for any $\alpha>1$ if and only if $\gamma>2$. Consequently, $\underline{\delta}^{C}<\underline{\delta}^{J}$ if and only if $\gamma>2$. Q.E.D. 


\section{Proof of Lemma 2.}

(i) The gain from deviation under ownership by agent $i$ is equal to

$$
\begin{aligned}
G^{i}= & \frac{1}{2}\left(\theta_{\ell}+\theta_{h}\right)\left(y_{i}^{i}+y^{*}\right)+\frac{1}{2}\left(\theta_{i}-\theta_{j}\right)\left(y_{i}^{i}+\mu y^{*}\right)-c\left(y_{i}^{i}\right)+\frac{1}{2}\left(\theta_{\ell}+\theta_{h}\right)\left(y^{*}+y_{j}^{i}\right) \\
& +\frac{1}{2}\left(\theta_{j}-\theta_{i}\right)\left(y^{*}+\mu y_{j}^{i}\right)-c\left(y_{j}^{i}\right)-2\left(\theta_{\ell}+\theta_{h}\right) y^{*}+2 c\left(y^{*}\right) \\
= & {\left[\theta_{i} y_{i}^{i}-c\left(y_{i}^{i}\right)\right]-\left[\theta_{i} y^{*}-c\left(y^{*}\right)\right] } \\
& +\left[\frac{1}{2}\left(\theta_{\ell}+\theta_{h}\right) y_{j}^{i}+\frac{1}{2}\left(\theta_{j}-\theta_{i}\right) \mu y_{j}^{i}-c\left(y_{j}^{i}\right)\right] \\
& -\left[\frac{1}{2}\left(\theta_{\ell}+\theta_{h}\right) y^{*}+\frac{1}{2}\left(\theta_{j}-\theta_{i}\right) \mu y^{*}-c\left(y^{*}\right)\right]
\end{aligned}
$$

Using the envelope theorem and taking into account that $\partial y^{*} / \partial \mu=0$, we obtain

$$
\begin{aligned}
& \frac{\partial G^{h}}{\partial \mu}=\frac{1}{2}\left(\theta_{\ell}-\theta_{h}\right)\left(y_{\ell}^{h}-y^{*}\right)>0 \\
& \frac{\partial G^{\ell}}{\partial \mu}=\frac{1}{2}\left(\theta_{h}-\theta_{\ell}\right)\left(y_{h}^{\ell}-y^{*}\right)<0 .
\end{aligned}
$$

Since $G^{h}=G^{\ell}=G^{C}$ for $\mu=1,(47)$ and (48) prove that $G^{h}<G^{C}<G^{\ell}$ for any $\mu<1$. According to Lemma $1 G^{J}<G^{C}$. Therefore $\max \left\{G^{J}, G^{h}\right\}<G^{C}<G^{\ell}$.

(ii) - (iii) (18) and (19) prove that $V^{h}<V^{C}<V^{\ell}$. Proposition 1 shows that $V^{C}<V^{J}$ if and only if $\gamma<1.5$ proving the statements in the Lemma. Q.E.D.

\section{Proof of Proposition 3.}

(i) According to Proposition $2 \underline{\delta}^{J}<\underline{\delta}^{C}$ if and only if $\gamma<2$. According to (14)-(17) $\underline{\delta}^{C}=\underline{\delta}^{h}=\underline{\delta}^{\ell}$ if $\mu=1$. Therefore for $\mu=1 \underline{\delta}^{J}<\min \left\{\underline{\delta}^{C}, \underline{\delta}^{h}, \underline{\delta}^{\ell}\right\}$ if $\gamma<2$. By continuity the same holds for $\mu \rightarrow 1$.

(ii) In the Online Appendix we prove that for $\mu=0 \underline{\delta}^{h}<\underline{\delta}^{J}<\underline{\delta}^{\ell}$ if and only if $\gamma<2$. According to Proposition $2 \underline{\delta}^{J}<\underline{\delta}^{C}$ if and only if $\gamma<2$. Therefore for $\mu=0$ $\underline{\delta}^{h}<\min \left\{\underline{\delta}^{C}, \underline{\delta}^{J}, \underline{\delta}^{\ell}\right\}$ if and only if $\gamma<2$. By continuity the same holds for $\mu \rightarrow 0$. 
(iii) According to Proposition $2 \underline{\delta}^{C}<\underline{\delta}^{J}$ if and only if $\gamma>2$. In the Online Appendix we prove that $\underline{\delta}^{C}<\min \left\{\underline{\delta}^{h}, \underline{\delta}^{\ell}\right\}$ if $\gamma>2$ and $\frac{\theta_{\ell}}{\theta_{h}} \rightarrow 0$. Therefore $\underline{\delta}^{C}<$ $\min \left\{\underline{\delta}^{J}, \underline{\delta}^{h}, \underline{\delta}^{\ell}\right\}$ if $\gamma>2$ and $\frac{\theta_{\ell}}{\theta_{h}} \rightarrow 0$.

(iv) In the Online Appendix we prove that for $\mu=0 \quad \underline{\delta}^{\ell}<\underline{\delta}^{J}<\underline{\delta}^{h}$ if and only if $\gamma>2$. According to Proposition $2 \underline{\delta}^{C}<\underline{\delta}^{J}$ if and only if $\gamma>2$. In the Online Appendix we prove that $\underline{\delta}^{\ell}<\underline{\delta}^{C}$ if $\gamma>2, \frac{\theta_{\ell}}{\theta_{h}} \rightarrow 1$ and $\mu \rightarrow 0$. Therefore $\underline{\delta}^{\ell}<\min \left\{\underline{\delta}^{J}, \underline{\delta}^{C}, \underline{\delta}^{h}\right\}$ if $\gamma>2, \frac{\theta_{\ell}}{\theta_{h}} \rightarrow 1$ and $\mu \rightarrow 0$. Q.E.D.

\section{A.2 Severe punishment investment}

If the agents are willing to engage in severe punishment $\left(y_{i}^{p}<y_{i}^{\omega}\right)$, the first-best investments can be sustained for lower discount factors than in Section 5. The maximum transfers are then

$$
F^{h, \omega}+F^{\ell, \omega}=\frac{S^{*}-P_{h}^{p, \omega}\left(y_{h}^{\omega}, y_{\ell}^{p}\right)-P_{\ell}^{p, \omega}\left(y_{\ell}^{\omega}, y_{h}^{p}\right)}{(1-\delta)} .
$$

$y_{i}^{p}$ has to be incentive compatible as determined by

$$
\begin{aligned}
& (1-\delta) P_{i}^{p, \omega}\left(y_{i}^{p}, y_{j}^{\omega}\right)+\delta\left[(1-\delta) F^{j, \omega}+P_{i}^{*}\left(y^{*}, y^{*}\right)\right] \\
\geq & (1-\delta) P_{i}^{p, \omega}\left(y_{i}^{\omega}, y_{j}^{\omega}\right)+\delta\left[-(1-\delta) F^{i, \omega}+P_{i}^{*}\left(y^{*}, y^{*}\right)\right]
\end{aligned}
$$

where $P_{i}^{*}\left(y^{*}, y^{*}\right)$ is agent $i$ 's payoff after cooperation is restored. (50) simplifies to

$$
\delta\left(F^{h, \omega}+F^{\ell, \omega}\right) \geq P_{i}^{p, \omega}\left(y_{i}^{\omega}, y_{j}^{\omega}\right)-P_{i}^{p, \omega}\left(y_{i}^{p}, y_{j}^{\omega}\right) .
$$


(51) is clearly satisfied for $y_{i}^{p}=y_{i}^{\omega}$. Then, consider a lower $y_{i}^{p}$ under joint ownership.

$$
\delta \frac{\partial\left(F^{h, J}+F^{\ell, J}\right)}{\partial y_{i}^{p}}+\frac{\partial P_{i}^{J}\left(y_{i}^{p}, y^{J}\right)}{\partial y_{i}^{p}}=\frac{1}{(1-\delta)}\left[(1-2 \delta) \frac{1}{2}\left(\theta_{\ell}+\theta_{h}\right)-(1-\delta) c^{\prime}\left(y_{i}^{p}\right)\right]
$$

(52) is negative for any $\delta \geq 1 / 2$ and therefore all $y_{i}^{p} \in\left[0, y^{J}\right]$ are incentive compatible. More severe punishment increases the maximum transfers more than the gain from deviating from $y_{i}^{p}$, relaxing the IC. We can verify that also under common ownership all $y_{i}^{p} \in\left[0, y_{i}^{C}\right]$ are incentive compatible if $\delta \geq 1 / 2$.

Finally, we check incentive compatibility of $y^{*}$ from (26) by taking into account that now $F^{h, \omega}+F^{\ell, \omega}$ is given by (49). Since $\partial F^{j, \omega} / \partial y_{i}^{p}<0$, it is optimal to set $y_{i}^{p}=0$. The critical discount factor is now

$$
\delta \geq \frac{P_{h}^{d, \omega}+P_{\ell}^{d, \omega}-S^{*}}{P_{h}^{d, \omega}+P_{\ell}^{d, \omega}-P_{h}^{p, \omega}\left(y_{h}^{\omega}, 0\right)-P_{\ell}^{p, \omega}\left(y_{\ell}^{\omega}, 0\right)}=\frac{G^{\omega}}{\left(\theta_{\ell}+\theta_{h}\right) y^{*}}
$$

By Proposition $2, G^{J}<G^{C}$. Therefore joint ownership dominates common ownership if $\delta \geq 1 / 2$. It can be shown that the same result holds also for $\delta<1 / 2$. 


\section{References}

[1] Aghion, Philippe and Jean Tirole. 1994. "The Management of Innovation." Quarterly Journal of Economics 109 (4): 1185-1209.

[2] Baker, George, Robert Gibbons and Kevin J. Murphy. 2002. "Relational Contracts and the Theory of the Firm." Quarterly Journal of Economics 117 (1): 39-84.

[3] Baland, Jean-Marie and Jean-Philippe Platteau. 2003. "Economics of Common Property Management Regimes." in Karl-Göran Mäler and Jeffrey R. Vincent (eds) Handbook of Environmental Economics, Volume 1, 127-190.

[4] Benhabib, Jess and Roy Radner. 1992. "Joint Exploitation of a Productive Asset." Economic Theory 2 (2): 155-190.

[5] Bennett, John and Elisabetta Iossa. 2006. "Building and Managing Facilities for Public Services." Journal of Public Economics 90 (10-11): 2143-2160.

[6] Besley, Timothy and Maitreesh Ghatak. 2001. "Government versus Private Ownership of Public Goods." Quarterly Journal of Economics 116 (4): 1343-72.

[7] Blonski, Matthias and Giancarlo Spagnolo. 2007. "Relational Efficient Property Rights." mimeo.

[8] Cai, Hongbin. 2003. "A Theory of Joint Asset Ownership." Rand Journal of Economics 34 (1): 63-77.

[9] Casari, Marco. 2007. "Emergence of Endogenous Legal Institutions: Property Rights and Community Governance in the Italian Alps." The Journal of Economic History 67 (1): 191-226. 
[10] Copeland, Brian R. and M. Scott Taylor. 2009. "Trade, Tragedy, and the Commons." American Economic Review 99 (3): 725-749.

[11] Coward, E. Walter and Robert Y. Siu. 1983. "Structuring Collective Action: An Irrigation Federation in the Northern Philippines." Philippine Sociological Review 31: $3-17$.

[12] Doornik, Katherine. 2006. "Relational Contracting in Partnerships." Journal of Economics and Management Strategy 15 (2): 517-548.

[13] Francesconi, Marco and Abhinay Muthoo. 2011. "Control Rights in Complex Partnerships." Journal of the European Economic Association 9 (3): 551-589.

[14] Goldlücke, Susanne and Sebastian Kranz. 2012. "Infinitely Repeated Games with Public Monitoring and Monetary Transfers." Journal of Economic Theory 147 (3): 1191-1221.

[15] Grossman, Sanford J. and Oliver D. Hart. 1986. "The Costs and Benefits of Ownership: A Theory of Vertical and Lateral Integration." Journal of Political Economy 94 (4): 691-719.

[16] Hagedoorn, John. 1996. "Trends and Patterns in Strategic Technology Partnering Since the Early Seventies." Review of Industrial Organization 11 (5): 601-616.

[17] Hagedoorn, John. 2002. "Inter-firm R\&D Partnerships - An Overview of Major Trends and Patterns Since 1960." Research Policy 31 (4): 477-492.

[18] Halac, Marina. 2012. "Relational Contracts and the Value of Relationships." American Economic Review 102 (2): 750-779.

[19] Halac, Marina. 2015. "Investing in a Relationship." RAND Journal of Economics 46 (1): 165-185. 
[20] Halonen, Maija. 2002. "Reputation and Allocation of Ownership." Economic Journal 112 (481): 539-558.

[21] Halonen-Akatwijuka, Maija. 2012. "Nature of Human Capital, Technology and Ownership of Public Goods." Journal of Public Economics 96 (11-12): 939-945.

[22] Harstad, Bård, Francesco Lancia and Alessia Russo. 2015. "Compliance Technology and Self-Enforcing Agreements." CESifo Working Paper 5562.

[23] Hart, Oliver. 1995. Firms, Contracts, and Financial Structure Oxford University Press.

[24] Hart, Oliver. 2003. "Incomplete Contracts and Public Ownership: Remarks and an Application to Public-Private Partnerships." Economic Journal 113 (486): C69-C76.

[25] Hart, Oliver. 2009 "Hold-up, Asset Ownership, and Reference Points." Quarterly Journal of Economics 124 (1): 267-300.

[26] Hart, Oliver and John Moore. 1990. "Property Rights and the Nature of the Firm." Journal of Political Economy 98 (6): 1119-1158.

[27] Hart, Oliver and John Moore. 2008. "Contracts as Reference Points." Quarterly Journal of Economics 123 (1): 1-48.

[28] Hart, Oliver, Andrei Shleifer and Robert W. Vishny. 1997. "The Proper Scope of Government: Theory and an Application to Prisons." Quarterly Journal of Economics 112 (4): 1127-1161.

[29] Levin, Jonathan. 2003. "Relational Incentive Contracts." American Economic Review 93 (3): 835-857. 
[30] Martin, Edward D. and Robert Yoder. 1988. "A Comparative Description of Two Farmer-Managed Irrigation Systems in Nepal." Irrigation and Drainage Systems $2(2): 147-172$.

[31] McKean, Margaret. 1982. "The Japanese Experience with Scarcity: Management of Traditional Common Lands." Environmental Review 6 (2): 63-91.

[32] Miller, David A. and Joel Watson. 2013. "A Theory of Disagreement in Repeated Games with Bargaining." Econometrica 81 (6): 2303-2350.

[33] Netting, Robert McC. 1972. "Of Men and Meadows: Strategies of Alpine Land Use." Anthropological Quarterly 45 (3): 132-144.

[34] Netting, Robert McC. 1976. "What Alpine Peasants Have in Common: Observations on Communal Tenure in a Swiss Village." Human Ecology 4 (2): 135-146.

[35] Niedermayer, Andras. 2013. "On Platforms, Incomplete Contracts, and Open Source Software." International Journal of Industrial Organization 31 (6): 714722 .

[36] Ostrom, Elinor. 1990. Governing the Commons: The Evolution of Institutions for Collective Actions Cambridge University Press.

[37] Ostrom, Elinor. 2002. "Common-pool Resources and Institutions: Toward a Revised Theory." in B. Gardner and G. Rausser (eds) Handbook of Agricultural Economics Volume 2. Elsevier Science B.V.

[38] Ostrom, Elinor and Roy Gardner. 1993. "Coping with Asymmetries in the Commons: Self-Governing Irrigation Systems Can Work." Journal of Economic Perspectives 7 (4): 93-112. 
[39] Ostrom, Elinor, Roy Gardner and James Walker. 1994. Rules, Games, and Common-Pool Resources University of Michigan Press.

[40] Ostrom, Elinor and Charlotte Hess. 2010. "Private and Common Property Rights." in Boudewin Bouckaert (ed.) Property Law and Economics Edward Elgar Publishing Limited.

[41] Poteete, Amy R. and Elinor Ostrom. 2004. "Heterogeneity, Group Size and Collective Action: The Role of Institutions in Forest Management." Development and Change 35 (3): 435-461.

[42] Ramey, Garey and Joel Watson, 1997. "Contractual Fragility, Job Destruction, and Business Cycles." Quarterly Journal of Economics 112 (3): 873-911.

[43] Rayo, Luis. 2007. "Relational Incentives and Moral Hazard in Teams." Review of Economic Studies 74 (3): 937-963.

[44] Rosenkranz, Stephanie and Patrick W. Schmitz. 2003. "Optimal Allocation of Ownership Rights in Dynamic R\&D Alliances." Games and Economic Behavior 43 (1): 153-173.

[45] Ruttan, Lore M. 2006. "Sociocultural Heterogeneity and the Commons." Current Anthropology 47 (5): 843-853.

[46] Ruttan, Lore M. 2008. "Economic Heterogeneity and the Commons: Effects on Collective Action and Collective Goods Provisioning." World Development 36 (5): 969-985.

[47] Stern, Paul C. 2011. "Design principles for global commons: natural resources and emerging technologies." International Journal of the Commons 5 (2): 213-232. 
[48] Tagliapietra, Claudio. 2011. "Charters, Partnerships and Natural Resources: Two Cases of Endogenous Regulation in Italy." Economic Affairs 31 (2): 30-35.

[49] Yoder, Robert. 1994. Organization and Management by Farmers in the Chhatis Mauja Irrigation System, Nepal. Colombo, Sri Lanka: IIMI 


\section{Online Appendix}

\section{for \\ Common Ownership of Public Goods}

Maija Halonen-Akatwijuka and Evagelos Pafilis

May 2018

\section{Proof of Proposition 1}

It remains to prove that $\partial^{2} \psi_{s}(\alpha, \gamma) / \partial \alpha^{2}>0$ for any $\alpha>1$ if and only if $\gamma>1.5$. Differentiating (35) we obtain

$$
\frac{\partial^{2} \psi_{s}(\alpha, \gamma)}{\partial \alpha^{2}}=\frac{1}{\gamma-1}\left[\frac{2 \gamma-1}{\gamma-1}\left(\frac{1}{2}\right)^{\frac{1}{\gamma-1}}(\alpha+1)^{\frac{2-\gamma}{\gamma-1}}-\alpha^{\frac{2-\gamma}{\gamma-1}}-\frac{2-\gamma}{\gamma-1} \alpha^{\frac{3-2 \gamma}{\gamma-1}}\right]
$$

Multiply by $(\gamma-1) / \alpha^{\frac{2-\gamma}{\gamma-1}}$ and denote the expression by $\varphi_{s}(\alpha, \gamma)$.

$$
\varphi_{s}(\alpha, \gamma)=\frac{2 \gamma-1}{\gamma-1}\left(\frac{1}{2}\right)^{\frac{1}{\gamma-1}}\left(\frac{\alpha+1}{\alpha}\right)^{\frac{2-\gamma}{\gamma-1}}-\frac{2-\gamma}{\gamma-1} \alpha^{-1}-1
$$

In what follows we prove that $\varphi_{s}(\alpha, \gamma)>0$ for any $\alpha>1$ if and only if $\gamma>1.5$.

Step 1. We prove that $\varphi_{s}(1, \gamma)>0$ if and only if $\gamma>1.5$.

Substitute $\alpha=1$ in (A.1). By standard calculations

$$
\varphi_{s}(1, \gamma)=\frac{2 \gamma-1}{2(\gamma-1)}-\frac{2-\gamma}{\gamma-1}-1>0 \text { if and only if } \gamma>1.5
$$

Step 2. We prove that $\lim _{\alpha \rightarrow \infty} \varphi_{s}(\alpha, \gamma)>0$ if and only if $\gamma>1.5$.

$\lim _{\alpha \rightarrow \infty} \varphi_{s}(\alpha, \gamma)=\frac{2 \gamma-1}{\gamma-1}\left(\frac{1}{2}\right)^{\frac{1}{\gamma-1}}-1>0$ if and only if $\rho_{1}(\gamma)>\tau_{1}(\gamma)$ where $\rho_{1}(\gamma)=(2 \gamma-1)\left(\frac{1}{2}\right)^{\frac{1}{\gamma-1}}$ and $\tau_{1}(\gamma)=(\gamma-1)$.

It is straightforward to establish that

$$
\begin{gathered}
\rho_{1}^{\prime}(\gamma)=\left(\frac{1}{2}\right)^{\frac{1}{\gamma-1}}\left[2-\frac{(2 \gamma-1)}{(\gamma-1)^{2}} \ln \left(\frac{1}{2}\right)\right]>0, \\
\rho_{1}^{\prime \prime}(\gamma)=\frac{1}{(\gamma-1)^{4}} \ln \left(\frac{1}{2}\right)\left(\frac{1}{2}\right)^{\frac{1}{\gamma-1}}\left[2(\gamma-1)+(2 \gamma-1) \ln \left(\frac{1}{2}\right)\right] .
\end{gathered}
$$

According to $\left(\right.$ A.2) $\rho_{1}^{\prime \prime}(\gamma)>0$ if and only if $\gamma<\frac{2+\ln \left(\frac{1}{2}\right)}{2+2 \ln \left(\frac{1}{2}\right)} \approx 2.1$. By standard calculations $\lim _{\gamma \rightarrow 1} \rho_{1}(\gamma)=\tau_{1}(1)=0, \rho_{1}(1.5)=\tau_{1}(1.5)=0.5$ and $\rho_{1}^{\prime}(1.5)>\tau_{1}^{\prime}(\gamma)=1$. 
Consider $\gamma<1$.5. Given $\rho_{1}(\gamma)$ is convex, $\tau_{1}(\gamma)$ is linear, and the above properties, it follows that $\rho_{1}(\gamma)<\tau_{1}(\gamma)$ for any $\gamma<1.5$.

Then, consider $\gamma>1.5$. $\rho_{1}(\gamma)$ is convex for $1.5<\gamma<\frac{2+\ln \left(\frac{1}{2}\right)}{2+2 \ln \left(\frac{1}{2}\right)}$ and concave for $\gamma>\frac{2+\ln \left(\frac{1}{2}\right)}{2+2 \ln \left(\frac{1}{2}\right)}$. Since $\lim _{\gamma \rightarrow \infty} \rho_{1}^{\prime}(\gamma)=2>\tau_{1}^{\prime}(\gamma)=1$, it follows that $\rho_{1}(\gamma)>\tau_{1}(\gamma)$ for any $\gamma>1.5$.

Therefore $\rho_{1}(\gamma)>\tau_{1}(\gamma)$ if and only if $\gamma>1$.5. Consequently, $\lim _{\alpha \rightarrow \infty} \varphi_{s}(\alpha, \gamma)>0$ if and only if $\gamma>1.5$.

Step 3. We prove that there exists a unique $\widetilde{\alpha}>1$ for which $\partial \varphi_{s}(\alpha, \gamma) /\left.\partial \alpha\right|_{\alpha=\widetilde{\alpha}}=0 . \varphi_{s}(\widetilde{\alpha}, \gamma)>$ 0 if and only if $\gamma>1.5$.

Differentiate $\varphi_{s}(\alpha, \gamma)$.

$$
\frac{\partial \varphi_{s}(\alpha, \gamma)}{\partial \alpha}=\frac{2-\gamma}{(\gamma-1) \alpha^{2}}\left[1-\frac{2 \gamma-1}{\gamma-1}\left(\frac{1}{2}\right)^{\frac{1}{\gamma-1}}\left(\frac{\alpha+1}{\alpha}\right)^{\frac{3-2 \gamma}{\gamma-1}}\right]
$$

Denote the term in the square brackets by $\nu_{s}(\alpha, \gamma)$. By standard calculations $\nu_{s}(1, \gamma)=1-\frac{2 \gamma-1}{4(\gamma-1)}>$ $0, \lim _{\alpha \rightarrow \infty} \nu_{s}(\alpha, \gamma)=1-\frac{2 \gamma-1}{\gamma-1}\left(\frac{1}{2}\right)^{\frac{1}{\gamma-1}}<0$ and $\partial \nu_{s}(\alpha, \gamma) / \partial \alpha=\frac{(2 \gamma-1)(3 \gamma-2)}{(\gamma-1)^{2} \alpha^{2}}\left(\frac{1}{2}\right)^{\frac{1}{\gamma-1}}\left(\frac{\alpha+1}{\alpha}\right)^{\frac{4-3 \gamma}{\gamma-1}}<0$ if and only if $\gamma>1.5$. Therefore there exists a unique $\widetilde{\alpha}>1$ for which $\nu_{s}(\widetilde{\alpha}, \gamma)=0$. This implies that $\partial \varphi_{s}(\alpha, \gamma) /\left.\partial \alpha\right|_{\alpha=\widetilde{\alpha}}=0$. Therefore

$$
\frac{2 \gamma-1}{\gamma-1}\left(\frac{1}{2}\right)^{\frac{1}{\gamma-1}}\left(\frac{\widetilde{\alpha}+1}{\widetilde{\alpha}}\right)^{\frac{3-2 \gamma}{\gamma-1}}=1 .
$$

Substitute $\widetilde{\alpha}$ in (A.1) and use (A.4).

$$
\begin{aligned}
\varphi_{s}(\widetilde{\alpha}, \gamma) & =\left[\frac{2 \gamma-1}{\gamma-1}\left(\frac{1}{2}\right)^{\frac{1}{\gamma-1}}\left(\frac{\widetilde{\alpha}+1}{\widetilde{\alpha}}\right)^{\frac{3-2 \gamma}{\gamma-1}}\right]\left(\frac{\widetilde{\alpha}+1}{\widetilde{\alpha}}\right)-\frac{2-\gamma}{\gamma-1} \widetilde{\alpha}^{-1}-1 \\
& =\frac{2 \gamma-3}{\widetilde{\alpha}(\gamma-1)}>0 \text { if and only if } \gamma>1.5
\end{aligned}
$$

Step 4. We have proved that $\varphi_{s}(1, \gamma)>0, \lim _{\alpha \rightarrow \infty} \varphi_{s}(\alpha, \gamma)>0$ and $\varphi_{s}(\widetilde{\alpha}, \gamma)>0$ if and only if $\gamma>1.5$. Consequently, $\varphi_{s}(\alpha, \gamma)>0$ for any $\alpha>1$ if and only if $\gamma>1.5$. Therefore, $\partial^{2} \psi_{s}(\alpha, \gamma) / \partial \alpha^{2}>0$ for any $\alpha>1$ if and only if $\gamma>1.5$. Q.E.D.

\section{Proof of Proposition 2}

\subsection{Proof of $\underline{\delta}^{C}<\underline{\delta}^{J} \Leftrightarrow \chi_{r}(\alpha, \gamma)<\psi_{r}(\alpha, \gamma)$}

From (40) and (43) $\underline{\delta}^{C}<\underline{\delta}^{J}$ if and only if

$$
\begin{gathered}
{\left[(\gamma-1)+(\gamma-1) \alpha^{\frac{\gamma}{\gamma-1}}-(\gamma-2)(\alpha+1)^{\frac{\gamma}{\gamma-1}}\right]\left[1-\left(\frac{1}{2}\right)^{\frac{1}{\gamma-1}}\right]<} \\
{\left[\left((\alpha+1)^{\frac{1}{\gamma-1}}-\alpha^{\frac{1}{\gamma-1}}\right)+\alpha\left((\alpha+1)^{\frac{1}{\gamma-1}}-1\right)\right]\left[(\gamma-1)\left(\frac{1}{2}\right)^{\frac{1}{\gamma-1}}-(\gamma-2)\right]}
\end{gathered}
$$




$$
\begin{gathered}
\Leftrightarrow\left[(\gamma-1)+(\gamma-1) \alpha^{\frac{\gamma}{\gamma-1}}\right]\left[1-\left(\frac{1}{2}\right)^{\frac{1}{\gamma-1}}\right]-(\alpha+1)^{\frac{\gamma}{\gamma-1}}\left(\frac{1}{2}\right)^{\frac{1}{\gamma-1}}< \\
\left(-\alpha-\alpha^{\frac{1}{\gamma-1}}\right)\left[(\gamma-1)\left(\frac{1}{2}\right)^{\frac{1}{\gamma-1}}-(\gamma-2)\right] \\
\Leftrightarrow(\gamma-1)\left[1-\left(\frac{1}{2}\right)^{\frac{1}{\gamma-1}}\right]+\alpha\left[(\gamma-1)\left(\frac{1}{2}\right)^{\frac{1}{\gamma-1}}-(\gamma-2)\right]< \\
\left(\frac{1}{2}\right)^{\frac{1}{\gamma-1}}(\alpha+1)^{\frac{\gamma}{\gamma-1}}-(\gamma-1) \alpha^{\frac{\gamma}{\gamma-1}}\left[1-\left(\frac{1}{2}\right)^{\frac{1}{\gamma-1}}\right]-\alpha^{\frac{1}{\gamma-1}}\left[(\gamma-1)\left(\frac{1}{2}\right)^{\frac{1}{\gamma-1}}-(\gamma-2)\right] \\
\Leftrightarrow \chi_{r}(\alpha, \gamma)<\psi_{r}(\alpha, \gamma)
\end{gathered}
$$

where

$$
\begin{gathered}
\chi_{r}(\alpha, \gamma)=(\gamma-1)\left[1-\left(\frac{1}{2}\right)^{\frac{1}{\gamma-1}}\right]+\alpha\left[(\gamma-1)\left(\frac{1}{2}\right)^{\frac{1}{\gamma-1}}-(\gamma-2)\right] \\
\psi_{r}(\alpha, \gamma)=\left(\frac{1}{2}\right)^{\frac{1}{\gamma-1}}(\alpha+1)^{\frac{\gamma}{\gamma-1}}-(\gamma-1) \alpha^{\frac{\gamma}{\gamma-1}}\left[1-\left(\frac{1}{2}\right)^{\frac{1}{\gamma-1}}\right]-\alpha^{\frac{1}{\gamma-1}}\left[(\gamma-1)\left(\frac{1}{2}\right)^{\frac{1}{\gamma-1}}-(\gamma-2)\right] .
\end{gathered}
$$

2.2 Proof of $\partial^{2} \psi_{r}(\alpha, \gamma) / \partial \alpha^{2}>0$ for any $\alpha>1$ if and only if $\gamma>2$

Differentiating (45) we obtain

$$
\begin{aligned}
\frac{\partial^{2} \psi_{r}(\alpha, \gamma)}{\partial \alpha^{2}}= & \frac{1}{\gamma-1}\left\{\frac{\gamma}{\gamma-1}\left(\frac{1}{2}\right)^{\frac{1}{\gamma-1}}(\alpha+1)^{\frac{2-\gamma}{\gamma-1}}-\gamma \alpha^{\frac{2-\gamma}{\gamma-1}}\left[1-\left(\frac{1}{2}\right)^{\frac{1}{\gamma-1}}\right]\right. \\
& \left.-\frac{2-\gamma}{\gamma-1} \alpha^{\frac{3-2 \gamma}{\gamma-1}}\left[(\gamma-1)\left(\frac{1}{2}\right)^{\frac{1}{\gamma-1}}-(\gamma-2)\right]\right\} .
\end{aligned}
$$

Multiply by $(\gamma-1) / \alpha^{\frac{2-\gamma}{\gamma-1}}$ and denote the expression by $\varphi_{r}(\alpha, \gamma)$.

$$
\begin{aligned}
\varphi_{r}(\alpha, \gamma)= & \alpha^{-1}\left\{\frac{\alpha \gamma}{\gamma-1}\left(\frac{1}{2}\right)^{\frac{1}{\gamma-1}}\left(\frac{\alpha+1}{\alpha}\right)^{\frac{2-\gamma}{\gamma-1}}-\gamma \alpha\left[1-\left(\frac{1}{2}\right)^{\frac{1}{\gamma-1}}\right]\right. \\
& \left.-\frac{2-\gamma}{\gamma-1}\left[(\gamma-1)\left(\frac{1}{2}\right)^{\frac{1}{\gamma-1}}-(\gamma-2)\right]\right\}
\end{aligned}
$$

In what follows we prove that $\varphi_{r}(\alpha, \gamma)>0$ for any $\alpha>1$ if and only if $\gamma>2$.

Step 1. We prove that $\varphi_{r}(1, \gamma)>0$ if and only if $\gamma>2$.

From (A.5)

$$
\varphi_{r}(1, \gamma)=\frac{\gamma}{2(\gamma-1)}+2(\gamma-1)\left(\frac{1}{2}\right)^{\frac{1}{\gamma-1}}+\frac{(2-\gamma)(\gamma-2)}{\gamma-1}-\gamma
$$


$\varphi_{r}(1, \gamma)>0$ if and only if

$$
\begin{gathered}
\frac{\gamma}{2(\gamma-1)}+\frac{(2-\gamma)(\gamma-1)}{\gamma-1}-\frac{2-\gamma}{\gamma-1}-\gamma>-2(\gamma-1)\left(\frac{1}{2}\right)^{\frac{1}{\gamma-1}} \\
\Leftrightarrow(2-\gamma)>\frac{\gamma}{4(\gamma-1)}-(\gamma-1)\left(\frac{1}{2}\right)^{\frac{1}{\gamma-1}}
\end{gathered}
$$

Denote $\tau_{2}(\gamma)=(2-\gamma)$ and $\rho_{2}(\gamma)=\frac{\gamma}{4(\gamma-1)}-(\gamma-1)\left(\frac{1}{2}\right)^{\frac{1}{\gamma-1}}$. It is straightforward to show that

$$
\begin{gathered}
\rho_{2}^{\prime}(\gamma)=-\frac{1}{4(\gamma-1)^{2}}-\left(\frac{1}{2}\right)^{\frac{1}{\gamma-1}}+\frac{1}{(\gamma-1)} \ln \left(\frac{1}{2}\right)\left(\frac{1}{2}\right)^{\frac{1}{\gamma-1}}<0, \\
\rho_{2}^{\prime \prime}(\gamma)=\frac{1}{2(\gamma-1)^{3}}\left[1-\left(\ln \left(\frac{1}{2}\right)\right)^{2}\left(\frac{1}{2}\right)^{\frac{2-\gamma}{\gamma-1}}\right]>0 .
\end{gathered}
$$

The term in the squared brackets is positive if and only if

$$
\gamma>\frac{2 \ln 2+\ln \left((\ln 2)^{2}\right)}{\ln 2+\ln \left((\ln 2)^{2}\right)} .
$$

The right-hand-side of $\left(\right.$ A.8) is negative since $\ln 2+\ln \left((\ln 2)^{2}\right)<0$. Therefore $\rho_{2}^{\prime \prime}(\gamma)>0$ for all $\gamma>1$.

By standard calculations $\tau_{2}(2)=\rho_{2}(2)=0, \rho_{2}^{\prime}(2)<\tau_{2}^{\prime}(\gamma)=-1$ and $\lim _{\gamma \rightarrow \infty} \rho_{2}^{\prime}(\gamma)=\tau_{2}^{\prime}(\gamma)=$ -1 . Since $\rho_{2}(\gamma)$ is convex and $\tau_{2}(\gamma)$ is linear, these properties imply that $\tau_{2}(\gamma)>\rho_{2}(\gamma)$ if and only if $\gamma>2$. Therefore $\varphi_{r}(1, \gamma)>0$ if and only if $\gamma>2$.

Step 2. We prove that $\lim _{\alpha \rightarrow \infty} \varphi_{r}(\alpha, \gamma)>0$ if and only if $\gamma>2$.

$\lim _{\alpha \rightarrow \infty} \varphi_{r}(\alpha, \gamma)=\gamma\left[\frac{\gamma}{\gamma-1}\left(\frac{1}{2}\right)^{\frac{1}{\gamma-1}}-1\right]>0$ if and only if $\rho_{3}(\gamma)>\tau_{3}(\gamma)$ where $\rho_{3}(\gamma)=\gamma\left(\frac{1}{2}\right)^{\frac{1}{\gamma-1}}$ and $\tau_{3}(\gamma)=(\gamma-1)$.

It is straightforward to establish that

$$
\begin{gathered}
\rho_{3}^{\prime}(\gamma)=\left(\frac{1}{2}\right)^{\frac{1}{\gamma-1}}\left[1-\frac{\gamma}{(\gamma-1)^{2}} \ln \left(\frac{1}{2}\right)\right]>0, \\
\rho_{3}^{\prime \prime}(\gamma)=\frac{1}{(\gamma-1)^{3}} \ln \left(\frac{1}{2}\right)\left(\frac{1}{2}\right)^{\frac{1}{\gamma-1}}\left[2+\frac{\gamma}{(\gamma-1)} \ln \left(\frac{1}{2}\right)\right]<0 .
\end{gathered}
$$

Note that according to $\left(\right.$ A.9) $\rho_{3}^{\prime \prime}(\gamma)<0$ if and only if $\gamma>2 /\left[2-\ln \left(\frac{1}{2}\right)\right]$. Since $2 /\left[2-\ln \left(\frac{1}{2}\right)\right]<1$ $\rho_{3}(\gamma)$ is concave in the relevant parameter range.

By standard calculations $\rho_{3}(2)=\tau_{3}(2)=1, \rho_{3}^{\prime}(2)>\tau_{3}^{\prime}(\gamma)=1$ and $\lim _{\gamma \rightarrow \infty} \rho_{3}^{\prime}(\gamma)=\tau_{3}^{\prime}(\gamma)=1$. Since $\rho_{3}(\gamma)$ is concave and $\tau_{3}(\gamma)$ is linear, these properties imply that $\rho_{3}(\gamma)>\tau_{3}(\gamma)$ if and only if $\gamma>2$. Therefore $\lim _{\alpha \rightarrow \infty} \varphi_{r}(\alpha, \gamma)>0$ if and only if $\gamma>2$.

Step 3. We prove that $\varphi_{r}(\alpha, \gamma)<0$ for all $\alpha>1$ if $\gamma<2$.

Assume $\gamma<2$. 
Differentiate $\varphi_{r}(\alpha, \gamma)$ with respect to $\alpha$.

$$
\frac{\partial \varphi_{r}(\alpha, \gamma)}{\partial \alpha}=\frac{2-\gamma}{\gamma-1} \alpha^{-2}\left[-\frac{\gamma}{\gamma-1}\left(\frac{1}{2}\right)^{\frac{1}{\gamma-1}}\left(\frac{\alpha+1}{\alpha}\right)^{\frac{3-2 \gamma}{\gamma-1}}+(\gamma-1)\left(\frac{1}{2}\right)^{\frac{1}{\gamma-1}}-(\gamma-2)\right]
$$

Denote the term in the square brackets by $\nu_{r}(\alpha, \gamma)$. Since $-\frac{\gamma}{\gamma-1}\left(\frac{1}{2}\right)^{\frac{1}{\gamma-1}}\left(\frac{\alpha+1}{\alpha}\right)^{\frac{3-2 \gamma}{\gamma-1}}<0$ and $(\gamma-1)\left(\frac{1}{2}\right)^{\frac{1}{\gamma-1}}-(\gamma-2)>0$ by (38), for a given $\gamma \neq 1.5$ there exists a unique $\widehat{\alpha}$, not necessarily greater than 1 , for which $\nu_{r}(\widehat{\alpha}, \gamma)=0$. Furthermore,

$$
\nu_{r}(1, \gamma)=-\frac{\gamma}{4(\gamma-1)}+(\gamma-1)\left(\frac{1}{2}\right)^{\frac{1}{\gamma-1}}-(\gamma-2)<0
$$

(A.6) determines the sign of (A.11) given $\gamma<2$.

Differentiate $\nu_{r}(\alpha, \gamma)$ with respect to $\alpha$.

$$
\frac{\partial \nu_{r}(\alpha, \gamma)}{\partial \alpha}=\frac{\gamma(3-2 \gamma)}{\alpha^{2}(\gamma-1)^{2}}\left(\frac{\alpha+1}{\alpha}\right)^{\frac{4-3 \gamma}{\gamma-1}}\left(\frac{1}{2}\right)^{\frac{1}{\gamma-1}}>0 \text { if and only if } \gamma<1.5
$$

(A.11) and (A.12) determine the sign of $\nu_{r}(\alpha, \gamma)$ as in the first column of Table 1. As per (A.10), $\frac{\partial \varphi_{r}(\alpha, \gamma)}{\partial \alpha}$ has the same sign as $\nu_{r}(\alpha, \gamma)$ since $\gamma<2$.

$$
\begin{array}{lll} 
& \nu_{r}(\alpha, \gamma) \text { and } \frac{\partial \varphi_{r}(\alpha, \gamma)}{\partial \alpha} & \varphi_{r}(\alpha, \gamma) \\
\gamma<1.5 & - \text { if and only if } \alpha<\widehat{\alpha} \text { where } \widehat{\alpha}>1 & - \\
1.5<\gamma<2 & - & -
\end{array}
$$

Table 1

Finally, we can determine the sign of $\varphi_{r}(\alpha, \gamma)$. From steps 1 and 2 we know that $\varphi_{r}(1, \gamma)<0$ and $\lim _{\alpha \rightarrow \infty} \varphi_{r}(\alpha, \gamma)<0$ since $\gamma<2$. Consider first $\gamma<1$.5. From Table 1 we know that $\varphi_{r}(\alpha, \gamma)$ is decreasing in $\alpha$ if and only if $\alpha<\widehat{\alpha}$. Consequently, $\varphi_{r}(\alpha, \gamma)<0$ for all $\alpha$ if $\gamma<1.5$. Then, consider $1.5<\gamma<2$. From Table 1 we know that $\varphi_{r}(\alpha, \gamma)$ is decreasing in $\alpha$ for all $\alpha>1$. Consequently, $\varphi_{r}(\alpha, \gamma)<0$ for all $\alpha$ if $1.5<\gamma<2$. Combining the two ranges verifies that $\varphi_{r}(\alpha, \gamma)<0$ for all $\alpha>1$ if $\gamma<2$.

The above properties are not sufficient to determine the sign of $\varphi_{r}(\alpha, \gamma)$ for $\gamma>2$. We therefore prove it differently in the next step.

Step 4. We prove that $\varphi_{r}(\alpha, \gamma)>0$ for all $\alpha>1$ if $\gamma>2$.

Assume $\gamma>2$.

By (A.5) $\varphi_{r}(\alpha, \gamma)>0$ if and only if

$$
\left[\frac{\alpha \gamma}{\gamma-1}\left(\frac{\alpha+1}{\alpha}\right)^{\frac{2-\gamma}{\gamma-1}}+\gamma \alpha-(2-\gamma)\right]\left(\frac{1}{2}\right)^{\frac{1}{\gamma-1}}-\frac{2-\gamma}{\gamma-1}>\gamma \alpha-(2-\gamma)
$$

Denote the left-hand-side of (A.13) by $\rho_{4}(\alpha, \gamma)$ and the right-hand-side by $\tau_{4}(\alpha, \gamma)$. 
Differentiate $\rho_{4}(\alpha, \gamma)$ with respect to $\alpha$.

$$
\frac{\partial \rho_{4}(\alpha, \gamma)}{\partial \alpha}=\left[\left(\frac{\alpha+1}{\alpha}\right)^{\frac{2-\gamma}{\gamma-1}} \frac{\gamma}{\gamma-1}\left(1+\frac{\gamma-2}{(\gamma-1)(\alpha+1)}\right)+\gamma\right]\left(\frac{1}{2}\right)^{\frac{1}{\gamma-1}}>0 \text { since } \gamma>2
$$

The second derivative is

$$
\frac{\partial^{2} \rho_{4}(\alpha, \gamma)}{\partial \alpha^{2}}=\frac{\gamma(\gamma-2)(2 \gamma-3)}{\alpha^{3}(\gamma-1)^{3}}\left(\frac{\alpha+1}{\alpha}\right)^{\frac{2-\gamma}{\gamma-1}-2}\left(\frac{1}{2}\right)^{\frac{1}{\gamma-1}}>0 \text { since } \gamma>2
$$

Next we establish that $\left.\frac{\partial \rho_{4}(\alpha, \gamma)}{\partial \alpha}\right|_{\alpha=1}>\left.\frac{\partial \tau_{4}(\alpha, \gamma)}{\partial \alpha}\right|_{\alpha=1}$.

$$
\begin{gathered}
{\left[2^{\frac{2-\gamma}{\gamma-1}} \frac{\gamma}{\gamma-1}\left(1+\frac{\gamma-2}{2(\gamma-1)}\right)+\gamma\right]\left(\frac{1}{2}\right)^{\frac{1}{\gamma-1}}>\gamma} \\
\Leftrightarrow(2-\gamma)>\frac{\gamma}{4(\gamma-1)}-(\gamma-1)\left(\frac{1}{2}\right)^{\frac{1}{\gamma-1}}
\end{gathered}
$$

(A.14) is equivalent to (A.6). Therefore, $\left.\frac{\partial \rho_{4}(\alpha, \gamma)}{\partial \alpha}\right|_{\alpha=1}>\left.\frac{\partial \tau_{4}(\alpha, \gamma)}{\partial \alpha}\right|_{\alpha=1}$ since $\gamma>2$.

We have shown that $\rho_{4}(1, \gamma)>\tau_{4}(1, \gamma)$ (in step 1) and $\left.\frac{\partial \rho_{4}(\alpha, \gamma)}{\partial \alpha}\right|_{\alpha=1}>\left.\frac{\partial \tau_{4}(\alpha, \gamma)}{\partial \alpha}\right|_{\alpha=1}=\gamma$. Since $\rho_{4}(\alpha, \gamma)$ is convex and $\tau_{4}(\alpha, \gamma)$ is linear in $\alpha$, these properties imply that $\rho_{4}(\alpha, \gamma)>\tau_{4}(\alpha, \gamma)$ for all $\alpha>1$ if $\gamma>2$. Therefore $\varphi_{r}(\alpha, \gamma)>0$ for all $\alpha>1$ if $\gamma>2$.

Step 5. Steps 3 and 4 establish that $\varphi_{r}(\alpha, \gamma)>0$ for any $\alpha>1$ if and only if $\gamma>2$. Consequently, $\partial^{2} \psi_{r}(\alpha, \gamma) / \partial \alpha^{2}>0$ for any $\alpha>1$ if and only if $\gamma>2$. Q.E.D. 


\section{Proof of Proposition 3}

\subsection{Derivation of $\underline{\delta}^{\ell}$ and $\underline{\delta}^{h}$}

We first work out $G^{i}$ and $G^{i}+V^{i}$ for $i=\ell, h$. From equation (46) $G^{i}$ equals

$$
\begin{aligned}
& G^{i}=\left[\theta_{i}\left(\frac{\theta_{i}}{\gamma}\right)^{\frac{1}{\gamma-1}}-\left(\frac{\theta_{i}}{\gamma}\right)^{\frac{\gamma}{\gamma-1}}\right]-\left[\theta_{i}\left(\frac{\theta_{\ell}+\theta_{h}}{\gamma}\right)^{\frac{1}{\gamma-1}}-\left(\frac{\theta_{\ell}+\theta_{h}}{\gamma}\right)^{\frac{\gamma}{\gamma-1}}\right] \\
& +\left[\frac{1}{2}\left[\left(\theta_{\ell}+\theta_{h}\right)+\left(\theta_{j}-\theta_{i}\right) \mu\right]\left(\frac{\theta_{\ell}+\theta_{h}+\mu\left(\theta_{j}-\theta_{i}\right)}{2 \gamma}\right)^{\frac{1}{\gamma-1}}-\left(\frac{\theta_{\ell}+\theta_{h}+\mu\left(\theta_{j}-\theta_{i}\right)}{2 \gamma}\right)^{\frac{\gamma}{\gamma-1}}\right] \\
& -\left[\frac{1}{2}\left[\left(\theta_{\ell}+\theta_{h}\right)+\left(\theta_{j}-\theta_{i}\right) \mu\right]\left(\frac{\theta_{\ell}+\theta_{h}}{\gamma}\right)^{\frac{1}{\gamma-1}}-\left(\frac{\theta_{\ell}+\theta_{h}}{\gamma}\right)^{\frac{\gamma}{\gamma-1}}\right] \\
& =\frac{1}{2}\left(\frac{1}{\gamma}\right)^{\frac{\gamma}{\gamma-1}}\left[2(\gamma-1)\left(\theta_{i}\right)^{\frac{\gamma}{\gamma-1}}-\gamma\left(2 \theta_{i}+\left(\theta_{j}-\theta_{i}\right) \mu\right)\left(\theta_{\ell}+\theta_{h}\right)^{\frac{1}{\gamma-1}}\right. \\
& \left.+(4-\gamma)\left(\theta_{\ell}+\theta_{h}\right)^{\frac{\gamma}{\gamma-1}}+(\gamma-1)\left(\frac{1}{2}\right)^{\frac{1}{\gamma-1}}\left(\theta_{\ell}+\theta_{h}+\mu\left(\theta_{j}-\theta_{i}\right)\right)^{\frac{\gamma}{\gamma-1}}\right] \\
& =\frac{1}{2}\left(\frac{1}{\gamma}\right)^{\frac{\gamma}{\gamma-1}}\left[2(\gamma-1)\left(\theta_{i}\right)^{\frac{\gamma}{\gamma-1}}+\gamma\left(\theta_{j}-\theta_{i}\right)(1-\mu)\left(\theta_{\ell}+\theta_{h}\right)^{\frac{1}{\gamma-1}}\right. \\
& \left.-2(\gamma-2)\left(\theta_{\ell}+\theta_{h}\right)^{\frac{\gamma}{\gamma-1}}+(\gamma-1)\left(\frac{1}{2}\right)^{\frac{1}{\gamma-1}}\left(\theta_{\ell}+\theta_{h}+\mu\left(\theta_{j}-\theta_{i}\right)\right)^{\frac{\gamma}{\gamma-1}}\right] \\
& G^{i}+V^{i}=\left[\theta_{i} y_{i}-c\left(y_{i}\right)\right]-\left[\theta_{i} y^{*}-c\left(y^{*}\right)\right]+\left[\frac{1}{2}\left[\left(\theta_{\ell}+\theta_{h}\right)+\left(\theta_{j}-\theta_{i}\right) \mu\right] y_{j}-c\left(y_{j}\right)\right] \\
& -\left[\frac{1}{2}\left[\left(\theta_{\ell}+\theta_{h}\right)+\left(\theta_{j}-\theta_{i}\right) \mu\right] y^{*}-c\left(y^{*}\right)\right] \\
& +2\left(\theta_{\ell}+\theta_{h}\right) y^{*}-2 c\left(y^{*}\right)-\left(\theta_{\ell}+\theta_{h}\right)\left(y_{i}^{i}+y_{j}^{i}\right)+c\left(y_{i}^{i}\right)+c\left(y_{j}^{i}\right) \\
& =\theta_{j}\left(y^{*}-y_{i}\right)+\frac{1}{2}\left[\left(\theta_{\ell}+\theta_{h}\right)-\left(\theta_{j}-\theta_{i}\right) \mu\right]\left(y^{*}-y_{j}\right) \\
& =\frac{1}{2}\left(\frac{1}{\gamma}\right)^{\frac{1}{\gamma-1}}\left\{2 \theta_{j}\left(\left(\theta_{\ell}+\theta_{h}\right)^{\frac{1}{\gamma-1}}-\left(\theta_{i}\right)^{\frac{1}{\gamma-1}}\right)\right. \\
& \left.+\left[\left(\theta_{\ell}+\theta_{h}\right)-\left(\theta_{j}-\theta_{i}\right) \mu\right]\left[\left(\theta_{\ell}+\theta_{h}\right)^{\frac{1}{\gamma-1}}-\left(\frac{1}{2}\right)^{\frac{1}{\gamma-1}}\left(\theta_{\ell}+\theta_{h}+\mu\left(\theta_{j}-\theta_{i}\right)\right)^{\frac{1}{\gamma-1}}\right]\right\}
\end{aligned}
$$

Substitute in $\theta_{j}=\beta \theta_{i}$. Note that $\beta \in[0,1)$ for $i=h$ and $\beta>1$ for $i=\ell$.

$$
\begin{aligned}
G^{i}= & \frac{1}{2}\left(\theta_{i}\right)^{\frac{\gamma}{\gamma-1}}\left(\frac{1}{\gamma}\right)^{\frac{\gamma}{\gamma-1}}\left[2(\gamma-1)+\gamma(\beta-1)(1-\mu)(\beta+1)^{\frac{1}{\gamma-1}}\right. \\
& \left.-2(\gamma-2)(\beta+1)^{\frac{\gamma}{\gamma-1}}+(\gamma-1)\left(\frac{1}{2}\right)^{\frac{1}{\gamma-1}}(\beta+1+\mu(\beta-1))^{\frac{\gamma}{\gamma-1}}\right]
\end{aligned}
$$




$$
\begin{aligned}
G^{i}+V^{i}= & \frac{1}{2}\left(\theta_{i}\right)^{\frac{\gamma}{\gamma-1}}\left(\frac{1}{\gamma}\right)^{\frac{1}{\gamma-1}}\left\{2 \beta\left((\beta+1)^{\frac{1}{\gamma-1}}-1\right)\right. \\
& \left.+[(\beta+1)-(\beta-1) \mu]\left[(\beta+1)^{\frac{1}{\gamma-1}}-\left(\frac{1}{2}\right)^{\frac{1}{\gamma-1}}(\beta+1+\mu(\beta-1))^{\frac{1}{\gamma-1}}\right]\right\}(A .1
\end{aligned}
$$

Using (A.17) and (A.18) we obtain $\underline{\delta}^{i}=G^{i} /\left(G^{i}+V^{i}\right)$.

\subsection{Proof of $\underline{\delta}^{h}<\underline{\delta}^{J}<\underline{\delta}^{\ell}$ if and only if $\gamma<2$ given $\mu=0$}

Step 1. We prove that for $\mu=0 \underline{\delta}^{i}<\underline{\delta}^{J} \Leftrightarrow \psi_{1}(\beta, \gamma)>\chi_{1}(\beta, \gamma)$

By substituting $\mu=0$ in (A.17) and (A.18) we obtain

$$
\underline{\delta}^{i}=\frac{2(\gamma-1)+\gamma(\beta-1)(\beta+1)^{\frac{1}{\gamma-1}}-2(\gamma-2)(\beta+1)^{\frac{\gamma}{\gamma-1}}+(\gamma-1)\left(\frac{1}{2}\right)^{\frac{1}{\gamma-1}}(\beta+1)^{\frac{\gamma}{\gamma-1}}}{\gamma\left[2 \beta\left((\beta+1)^{\frac{1}{\gamma-1}}-1\right)+(\beta+1)^{\frac{\gamma}{\gamma-1}}\left(1-\left(\frac{1}{2}\right)^{\frac{1}{\gamma-1}}\right)\right]} .
$$

According to (A.19) and (40) $\underline{\delta}^{i}<\underline{\delta}^{J}$ is equivalent to

$$
\begin{gathered}
{\left[2(\gamma-1)+\gamma(\beta-1)(\beta+1)^{\frac{1}{\gamma-1}}-2(\gamma-2)(\beta+1)^{\frac{\gamma}{\gamma-1}}+(\gamma-1)\left(\frac{1}{2}\right)^{\frac{1}{\gamma-1}}(\beta+1)^{\frac{\gamma}{\gamma-1}}\right]\left(1-\left(\frac{1}{2}\right)^{\frac{1}{\gamma-1}}\right)} \\
\quad<\left[2 \beta\left((\beta+1)^{\frac{1}{\gamma-1}}-1\right)+(\beta+1)^{\frac{\gamma}{\gamma-1}}\left(1-\left(\frac{1}{2}\right)^{\frac{1}{\gamma-1}}\right)\right]\left[(\gamma-1)\left(\frac{1}{2}\right)^{\frac{1}{\gamma-1}}-(\gamma-2)\right] \\
\Leftrightarrow(\beta+1)^{\frac{1}{\gamma-1}}\left[\beta-(1-\gamma(1-\beta))\left(1-\left(\frac{1}{2}\right)^{\frac{1}{\gamma-1}}\right)\right]>(1-\beta)(\gamma-1)\left(1-\left(\frac{1}{2}\right)^{\frac{1}{\gamma-1}}\right)+\beta \text { (A.20) }
\end{gathered}
$$

Denote

$$
\begin{gathered}
\psi_{1}(\beta, \gamma)=(\beta+1)^{\frac{1}{\gamma-1}}\left[\beta-(1-\gamma(1-\beta))\left(1-\left(\frac{1}{2}\right)^{\frac{1}{\gamma-1}}\right)\right] \\
\chi_{1}(\beta, \gamma)=(1-\beta)(\gamma-1)\left(1-\left(\frac{1}{2}\right)^{\frac{1}{\gamma-1}}\right)+\beta
\end{gathered}
$$

Therefore $\underline{\delta}^{i}<\underline{\delta}^{J}$ if and only if $\psi_{1}(\beta, \gamma)>\chi_{1}(\beta, \gamma)$. Note that $\underline{\delta}^{h}<\underline{\delta}^{J} \Leftrightarrow \psi_{1}(\beta, \gamma)>\chi_{1}(\beta, \gamma)$ where $\beta \in[0,1)$ and $\underline{\delta}^{\ell}<\underline{\delta}^{J} \Leftrightarrow \psi_{1}(\beta, \gamma)>\chi_{1}(\beta, \gamma)$ where $\beta>1$.

Step 2.We prove that $\psi_{1}(0, \gamma)=\chi_{1}(0, \gamma)$ and $\psi_{1}(1, \gamma)=\chi_{1}(1, \gamma)$.

By standard calculations $\psi_{1}(0, \gamma)=\chi_{1}(0, \gamma)=(\gamma-1)\left(1-\left(\frac{1}{2}\right)^{\frac{1}{\gamma-1}}\right)$ and $\psi_{1}(1, \gamma)=\chi_{1}(1, \gamma)=$ 1 .

Step 3. We prove that $(i) \frac{\partial \chi_{1}(\beta, \gamma)}{\partial \beta}>0$, (ii) $\left.\frac{\partial \psi_{1}(\beta, \gamma)}{\partial \beta}\right|_{\beta=0}=\left.\frac{\partial \chi_{1}(\beta, \gamma)}{\partial \beta}\right|_{\beta=0}$, (iii) $\frac{\partial \psi_{1}(\beta, \gamma)}{\partial \beta}>0$ if $\gamma>2$, and (iv) for $\gamma<2 \frac{\partial \psi_{1}(\beta, \gamma)}{\partial \beta}>0$ if and only if $\beta<\widetilde{\beta}$. 
Differentiating with respect to $\beta$ gives

$$
\begin{gathered}
\frac{\partial \chi_{1}(\beta, \gamma)}{\partial \beta}=(\gamma-1)\left(\frac{1}{2}\right)^{\frac{1}{\gamma-1}}-(\gamma-2)>0 \\
\frac{\partial \psi_{1}(\beta, \gamma)}{\partial \beta}=\frac{1}{\gamma-1}(\beta+1)^{\frac{1}{\gamma-1}-1}\left[-(\gamma-1)(\gamma-2+\beta \gamma)+\left(\frac{1}{2}\right)^{\frac{1}{\gamma-1}}\left((\gamma-1)^{2}+\beta \gamma^{2}\right)\right] .
\end{gathered}
$$

$\frac{\partial \chi_{1}(\beta, \gamma)}{\partial \beta}>0$ by (38). Furthermore,

$$
\left.\frac{\partial \psi_{1}(\beta, \gamma)}{\partial \beta}\right|_{\beta=0}=-(\gamma-2)+(\gamma-1)\left(\frac{1}{2}\right)^{\frac{1}{\gamma-1}}=\frac{\partial \chi_{1}(\beta, \gamma)}{\partial \beta}
$$

$\frac{\partial \psi_{1}(\beta, \gamma)}{\partial \beta}>0$ if and only if

$$
\begin{gathered}
-(\gamma-1)(\gamma-2+\beta \gamma)+\left(\frac{1}{2}\right)^{\frac{1}{\gamma-1}}\left[(\gamma-1)^{2}+\beta \gamma^{2}\right]>0 \\
\Leftrightarrow(\gamma-1)\left[(\gamma-1)\left(\frac{1}{2}\right)^{\frac{1}{\gamma-1}}-(\gamma-2)\right]>\beta \gamma\left[(\gamma-1)-\gamma\left(\frac{1}{2}\right)^{\frac{1}{\gamma-1}}\right]
\end{gathered}
$$

The left-hand side of (A.21) is positive by (38). The right-hand-side is negative if and only if $\gamma>2$ as verified in step 2 of the proof of Proposition 2. Therefore, (A.21) is satisfied if $\gamma>2$. Consequently, $\frac{\partial \psi_{1}(\beta, \gamma)}{\partial \beta}>0$ if $\gamma>2$. For $\gamma<2 \frac{\partial \psi_{1}(\beta, \gamma)}{\partial \beta}>0$ if and only if

$$
\beta<\frac{(\gamma-1)\left[\left(\frac{1}{2}\right)^{\frac{1}{\gamma-1}}(\gamma-1)-(\gamma-2)\right]}{\gamma\left[(\gamma-1)-\gamma\left(\frac{1}{2}\right)^{\frac{1}{\gamma-1}}\right]} \equiv \widetilde{\beta}>0
$$

Step 4. We prove that $(i)$ for $\gamma<2 \frac{\partial \psi_{1}^{2}(\beta, \gamma)}{\partial \beta^{2}}>0$ if and only if $\beta<\widehat{\beta}$, and (ii) for $\gamma>2 \frac{\partial \psi_{1}^{2}(\beta, \gamma)}{\partial \beta^{2}}>0$ if and only if $\beta>\widehat{\beta}$.

$$
\frac{\partial \psi_{1}^{2}(\beta, \gamma)}{\partial \beta^{2}}=\frac{1}{(\gamma-1)^{2}}(\beta+1)^{\frac{1}{\gamma-1}-2}\left\{-(\gamma-1)[\gamma(\beta+1)-2(2-\gamma)]+\left[\gamma^{2}(\beta+1)+2(\gamma-1)^{2}-\gamma\right]\left(\frac{1}{2}\right)^{\frac{1}{\gamma-1}}\right\}
$$

$\frac{\partial \psi_{1}^{2}(\beta, \gamma)}{\partial \beta^{2}}>0$ if and only if the term in the curly brackets is positive, i.e. if and only if

$$
-(\gamma-1)(\gamma-2(2-\gamma))+\left[\gamma^{2}+2(\gamma-1)^{2}-\gamma\right]\left(\frac{1}{2}\right)^{\frac{1}{\gamma-1}}>\gamma \beta\left[(\gamma-1)-\gamma\left(\frac{1}{2}\right)^{\frac{1}{\gamma-1}}\right] .
$$

The right-hand-side of (A.22) is positive if and only if $\gamma<2$. Consequently, for $\gamma<2 \frac{\partial^{2} \psi_{1}(\beta, \gamma)}{\partial \beta^{2}}>0$ if and only if

$$
\beta<\frac{(\gamma-1)\left[(3 \gamma-2)\left(\frac{1}{2}\right)^{\frac{1}{\gamma-1}}-(3 \gamma-4)\right]}{\gamma\left[(\gamma-1)-\gamma\left(\frac{1}{2}\right)^{\frac{1}{\gamma-1}}\right]} \equiv \widehat{\beta}
$$


For $\gamma>2$ the right-hand-side of (A.22) is negative and, therefore, $\frac{\partial^{2} \psi_{1}(\beta, \gamma)}{\partial \beta^{2}}>0$ if and only if $\beta>\widehat{\beta}$.

Step 5. We prove that $(i) \widehat{\beta}>0$ and (ii) $\widehat{\beta}<\widetilde{\beta}$ if $\gamma<2$.

First, we show that $(3 \gamma-2)\left(\frac{1}{2}\right)^{\frac{1}{\gamma-1}}-(3 \gamma-4)>0$ if and only if $\gamma<2$. Denote $\tau_{5}(\gamma)=(3 \gamma-4)$ and $\rho_{5}(\gamma)=(3 \gamma-2)\left(\frac{1}{2}\right)^{\frac{1}{\gamma-1}}$. By standard calculations

$$
\begin{gathered}
\rho_{5}^{\prime}(\gamma)=\left(\frac{1}{2}\right)^{\frac{1}{\gamma-1}}\left[3-\frac{(3 \gamma-2)}{(\gamma-1)^{2}} \ln \left(\frac{1}{2}\right)\right] \\
\rho_{5}^{\prime \prime}(\gamma)=\frac{1}{(\gamma-1)^{3}} \ln \left(\frac{1}{2}\right)\left(\frac{1}{2}\right)^{\frac{1}{\gamma-1}}\left[2+\frac{(3 \gamma-2)}{(\gamma-1)} \ln \left(\frac{1}{2}\right)\right]
\end{gathered}
$$

Note that $\rho_{5}^{\prime \prime}(\gamma)>0$ if and only if $\gamma>\frac{2+2 \ln \left(\frac{1}{2}\right)}{2+3 \ln \left(\frac{1}{2}\right)}$. Since $2+3 \ln \left(\frac{1}{2}\right)<0, \rho_{5}^{\prime \prime}(\gamma)>0$ for all $\gamma>1$.

By standard calculations $\tau_{5}(2)=\rho_{5}(2)=2, \tau_{5}^{\prime}(2)>\rho_{5}^{\prime}(2)>0$ and $\lim _{\gamma \rightarrow \infty} \rho_{5}^{\prime}(\gamma)=\tau_{5}^{\prime}(\gamma)=3$. Since $\rho_{5}(\gamma)$ is convex and and $\tau_{5}(\gamma)$ is linear, these properties imply that $\tau_{5}(\gamma)<\rho_{5}(\gamma)$ if and only if $\gamma<2$. Consequently, $(3 \gamma-2)\left(\frac{1}{2}\right)^{\frac{1}{\gamma-1}}-(3 \gamma-4)>0$ if and only if $\gamma<2$. Therefore the numerator and denominator of (A.23) have the same sign and, consequently, $\widehat{\beta}>0$.

Second, we prove that $\widehat{\beta}<\widetilde{\beta}$ if $\gamma<2$ by contradiction. Suppose $\widetilde{\beta}<\widehat{\beta}$ and consider $\beta \in(0, \widehat{\beta}]$. Then, from steps 3 and 4 , if $\gamma<2 \psi_{1}(\beta, \gamma)$ is increasing in $\beta$ for $\beta \in(0, \tilde{\beta}]$ and decreasing in $\beta$ for $\beta \in(\widetilde{\beta}, \widehat{\beta}]$ contradicting that $\psi_{1}(\beta, \gamma)$ is convex in the range of $\beta \in(0, \widehat{\beta})$. Consequently, $\widehat{\beta}<\widetilde{\beta}$ if $\gamma<2$.

Step 6. We prove that $\psi_{1}(\beta, \gamma)<\chi_{1}(\beta, \gamma)$ if and only if $(\gamma-2)(1-\beta)>0$.

Consider first $\gamma>2$. According to steps $2-5 \psi_{1}(0, \gamma)=\chi_{1}(0, \gamma), \psi_{1}(1, \gamma)=\chi_{1}(1, \gamma), \psi_{1}(\beta, \gamma)$ is increasing and concave in $\beta$ for $\beta \in(0, \widehat{\beta}]$ and increasing and convex in $\beta$ for $\beta>\widehat{\beta}$. Since $\left.\frac{\partial \psi_{1}(\beta, \gamma)}{\partial \beta}\right|_{\beta=0}=\left.\frac{\partial \chi_{1}(\beta, \gamma)}{\partial \beta}\right|_{\beta=0}$ and $\chi_{1}(\beta, \gamma)$ is linear in $\beta$, it follows that $\psi_{1}(\beta, \gamma)=\chi_{1}(\beta, \gamma)$ only for $\beta=0$ and $\beta=1$. Consequently, $\psi_{1}(\beta, \gamma)<\chi_{1}(\beta, \gamma)$ if and only if $\beta<1$.

Then, consider $\gamma<2$. According to steps $2-5 \psi_{1}(0, \gamma)=\chi_{1}(0, \gamma), \psi_{1}(1, \gamma)=\chi_{1}(1, \gamma), \psi_{1}(\beta, \gamma)$ is increasing and convex in $\beta$ for $\beta \in(0, \widehat{\beta}]$, increasing and concave in $\beta$ for $\beta \in(\widehat{\beta}, \widetilde{\beta}]$ and decreasing and concave in $\beta$ for $\beta>\widetilde{\beta}$. Since $\left.\frac{\partial \psi_{1}(\beta, \gamma)}{\partial \beta}\right|_{\beta=0}=\left.\frac{\partial \chi_{1}(\beta, \gamma)}{\partial \beta}\right|_{\beta=0}$ it follows that $\psi_{1}(\beta, \gamma)=\chi_{1}(\beta, \gamma)$ only for $\beta=0$ and $\beta=1$. Therefore $\psi_{1}(\beta, \gamma)<\chi_{1}(\beta, \gamma)$ if and only if $\beta>1$.

Step 7. We prove that for $\mu=0 \underline{\delta}^{h}<\underline{\delta}^{J}<\underline{\delta}^{\ell}$ if and only if $\gamma<2$.

By definition $\underline{\delta}^{J}<\underline{\delta}^{h} \Leftrightarrow \psi_{1}(\beta, \gamma)<\chi_{1}(\beta, \gamma)$ where $\beta \in[0,1)$. Step 6 shows that $\psi_{1}(\beta, \gamma)<$ $\chi_{1}(\beta, \gamma)$ for $\beta \in[0,1)$ if and only if $\gamma>2$. Similarly, by definition $\underline{\delta}^{J}<\underline{\delta}^{\ell} \Leftrightarrow \psi_{1}(\beta, \gamma)<\chi_{1}(\beta, \gamma)$ where $\beta>1$. Step 6 shows that $\psi_{1}(\beta, \gamma)<\chi_{1}(\beta, \gamma)$ for $\beta>1$ if and only if $\gamma<2$. Consequently, for $\mu=0 \underline{\delta}^{\ell}<\underline{\delta}^{J}<\underline{\delta}^{h}$ if and only if $\gamma>2$. Q.E.D.

\subsection{Proof of $\underline{\delta}^{C}<\min \left\{\underline{\delta}^{h}, \underline{\delta}^{\ell}\right\}$ if $\gamma>2$ and $\frac{\theta_{\ell}}{\theta_{h}} \rightarrow 0$}

Step 1. We derive $\underline{\delta}^{\ell}, \underline{\delta}^{h}$ and $\underline{\delta}^{C}$ for $\frac{\theta_{\ell}}{\theta_{h}}=0$. 
Substituting $\theta_{\ell}=0$ in (A.15) and (A.16) we obtain

$$
\begin{gathered}
\underline{\delta}^{\ell}=\frac{\gamma(1-\mu)-2(\gamma-2)+(\gamma-1)\left(\frac{1}{2}\right)^{\frac{1}{\gamma-1}}(1+\mu)^{\frac{\gamma}{\gamma-1}}}{\gamma\left[2+(1-\mu)\left(1-\left(\frac{1}{2}\right)^{\frac{1}{\gamma-1}}(1+\mu)^{\frac{1}{\gamma-1}}\right)\right]}, \\
\underline{\delta}^{h}=\frac{2-\gamma(1-\mu)+(\gamma-1)\left(\frac{1}{2}\right)^{\frac{1}{\gamma-1}}(1-\mu)^{\frac{\gamma}{\gamma-1}}}{\gamma(1+\mu)\left(1-\left(\frac{1}{2}\right)^{\frac{1}{\gamma-1}}(1-\mu)^{\frac{1}{\gamma-1}}\right)} .
\end{gathered}
$$

Substituting $\theta_{\ell}=0$ in (41) and (42) we obtain

$$
\underline{\delta}^{C}=\frac{1}{\gamma}
$$

Step 2. We prove that $\underline{\delta}^{C}<\underline{\delta}^{\ell}$ if $\gamma>2$ and $\frac{\theta_{\ell}}{\theta_{h}} \rightarrow 0$.

Assume $\gamma>2$. According to (A.24) and (A.26) $\underline{\delta}^{C}<\underline{\delta}^{\ell}$ is equivalent to

$$
\begin{gathered}
2+(1-\mu)\left(1-\left(\frac{1}{2}\right)^{\frac{1}{\gamma-1}}(1+\mu)^{\frac{1}{\gamma-1}}\right)<\gamma(1-\mu)-2(\gamma-2)+(\gamma-1)\left(\frac{1}{2}\right)^{\frac{1}{\gamma-1}}(1+\mu)^{\frac{\gamma}{\gamma-1}} \\
\Leftrightarrow[(\gamma-1)(1+\mu)+(1-\mu)]\left(\frac{1}{2}\right)^{\frac{1}{\gamma-1}}(1+\mu)^{\frac{1}{\gamma-1}}-(\gamma-1)(1+\mu)>0
\end{gathered}
$$

Denote the left-hand-side of (A.27) by $\phi_{\ell}(\mu, \gamma) . \phi_{\ell}(0, \gamma)=\gamma\left(\frac{1}{2}\right)^{\frac{1}{\gamma-1}}-(\gamma-1)>0$ since $\gamma>2$ (by step 2 of proof of Proposition 2) and $\phi_{\ell}(1, \gamma)=0$.

Differentiate $\phi_{\ell}(\mu, \gamma)$ with respect to $\mu$.

$$
\frac{\partial \phi_{\ell}(\mu, \gamma)}{\partial \mu}=(\gamma-2)\left(\frac{1}{2}\right)^{\frac{1}{\gamma-1}}(1+\mu)^{\frac{1}{\gamma-1}}+\frac{1}{\gamma-1}[(\gamma-1)(1+\mu)+(1-\mu)]\left(\frac{1}{2}\right)^{\frac{1}{\gamma-1}}(1+\mu)^{\frac{2-\gamma}{\gamma-1}}-(\gamma-1)
$$

Note that $\left.\frac{\partial \phi_{\ell}(\mu, \gamma)}{\partial \mu}\right|_{\mu=1}=0$.

Furthermore,

$$
\frac{\partial^{2} \phi_{\ell}(\mu, \gamma)}{\partial \mu^{2}}=\left(\frac{\gamma-2}{\gamma-1}\right)\left(\frac{1}{2}\right)^{\frac{1}{\gamma-1}}(1+\mu)^{\frac{2-\gamma}{\gamma-1}}\left[\frac{(\gamma-2)+\gamma \mu}{(\gamma-1)(1+\mu)}\right]>0 \text { since } \gamma>2
$$

We have shown that $\phi_{\ell}(0, \gamma)>0, \phi_{\ell}(1, \gamma)=0,\left.\frac{\partial \phi_{\ell}(\mu, \gamma)}{\partial \mu}\right|_{\mu=1}=0$ and $\frac{\partial^{2} \phi_{\ell}(\mu, \gamma)}{\partial \mu^{2}}>0$. Therefore $\frac{\partial \phi_{\ell}(\mu, \gamma)}{\partial \mu}<0$ for all $\mu \in[0,1)$. Consequently, $\phi_{\ell}(\mu, \gamma)>0$ for all $\mu \in[0,1)$. This proves that $\underline{\delta}^{C}<\underline{\delta}^{\ell}$ if $\frac{\theta_{\ell}}{\theta_{h}}=0$ and $\gamma>2$. By continuity the same holds for $\frac{\theta_{\ell}}{\theta_{h}} \rightarrow 0$ and $\gamma>2$.

Step 3. We prove that $\underline{\delta}^{C}<\underline{\delta}^{h}$ if $\gamma>2$ and $\frac{\theta_{\ell}}{\theta_{h}} \rightarrow 0$.

Assume $\gamma>2$. From (A.25) and (A.26) $\underline{\delta}^{C}<\underline{\delta}^{h}$ if and only if

$$
(1+\mu)-\left(\frac{1}{2}\right)^{\frac{1}{\gamma-1}}(1+\mu)(1-\mu)^{\frac{1}{\gamma-1}}<2-\gamma(1-\mu)+(\gamma-1)\left(\frac{1}{2}\right)^{\frac{1}{\gamma-1}}(1-\mu)^{\frac{\gamma}{\gamma-1}}
$$




$$
\Leftrightarrow[(\gamma-1)(1-\mu)+(1+\mu)]\left(\frac{1}{2}\right)^{\frac{1}{\gamma-1}}(1-\mu)^{\frac{1}{\gamma-1}}-(\gamma-1)(1-\mu)>0
$$

Denote the left-hand-side of (A.29) by $\phi_{h}(\mu, \gamma)$. Note that $\phi_{h}(0, \gamma)=\gamma\left(\frac{1}{2}\right)^{\frac{1}{\gamma-1}}-(\gamma-1)>0$ since $\gamma>2$ and $\phi_{h}(1, \gamma)=0$.

$$
\frac{\partial \phi_{h}(\mu, \gamma)}{\partial \mu}=(2-\gamma)\left(\frac{1}{2}\right)^{\frac{1}{\gamma-1}}(1-\mu)^{\frac{1}{\gamma-1}}-\frac{1}{\gamma-1}[(\gamma-1)(1-\mu)+(1+\mu)]\left(\frac{1}{2}\right)^{\frac{1}{\gamma-1}}(1-\mu)^{\frac{2-\gamma}{\gamma-1}}+(\gamma-1)
$$

Note that $\left.\frac{\partial \phi_{h}(\mu, \gamma)}{\partial \mu}\right|_{\mu \rightarrow 1} \rightarrow-\infty$ since $\gamma>2$. Furthermore,

$$
\left.\frac{\partial \phi_{h}(\mu, \gamma)}{\partial \mu}\right|_{\mu=0}=(2-\gamma)\left(\frac{1}{2}\right)^{\frac{1}{\gamma-1}}-\frac{\gamma}{\gamma-1}\left(\frac{1}{2}\right)^{\frac{1}{\gamma-1}}+(\gamma-1)
$$

$\left.\frac{\partial \phi_{h}(\mu, \gamma)}{\partial \mu}\right|_{\mu=0}>0$ if and only if

$$
1>\frac{1}{(\gamma-1)}\left[\frac{\gamma}{\gamma-1}-(2-\gamma)\right]\left(\frac{1}{2}\right)^{\frac{1}{\gamma-1}}
$$

Denote the right-hand-side of (A.30) by $\rho_{6}(\gamma)$.

$$
\rho_{6}^{\prime}(\gamma)=\frac{1}{(\gamma-1)^{4}}\left(\frac{1}{2}\right)^{\frac{1}{\gamma-1}}\left[-2(\gamma-1)-(\gamma-(2-\gamma)(\gamma-1)) \ln \left(\frac{1}{2}\right)\right]
$$

It is straightforward to show that $\rho_{6}^{\prime}(\gamma)=0$ for $\gamma_{1} \approx 1.4$ and $\gamma_{2} \approx 3.48$. Moreover, $\rho_{6}^{\prime}(2)<0$. Therefore, $\rho_{6}^{\prime}(\gamma)<0$ for $2 \leq \gamma<\gamma_{2}$ and $\rho_{6}^{\prime}(\gamma)>0$ for $\gamma>\gamma_{2}$. Note that $\rho_{6}(2)=1, \rho_{6}\left(\gamma_{2}\right)=$ 0.88 and $\lim _{\gamma \rightarrow \infty} \rho_{6}(\gamma)=1$. The above properties imply that for $\gamma>2 \rho_{6}(\gamma)<1$ and thus $\left.\frac{\partial \phi_{h}(\mu, \gamma)}{\partial \mu}\right|_{\mu=0}>0$.

By standard calculations

$$
\frac{\partial^{2} \phi_{h}(\mu, \gamma)}{\partial \mu^{2}}=\frac{\gamma-2}{\gamma-1}\left(\frac{1}{2}\right)^{\frac{1}{\gamma-1}}(1-\mu)^{\frac{2-\gamma}{\gamma-1}}\left[\frac{(\gamma-2)-\gamma \mu}{(\gamma-1)(1-\mu)}\right]>0
$$

if and only if $\mu<(\gamma-2) / \gamma \equiv \widetilde{\mu}$.

We have shown that $\phi_{h}(0, \gamma)>0,\left.\frac{\partial \phi_{h}(\mu, \gamma)}{\partial \mu}\right|_{\mu=0}>0$ and $\frac{\partial^{2} \phi_{h}(\mu, \gamma)}{\partial \mu^{2}}>0$ for all $\mu \in[0, \widetilde{\mu}]$. Therefore $\phi_{h}(\mu, \gamma)>0$ for all $\mu \in[0, \widetilde{\mu}]$. Furthermore, $\phi_{h}(\mu, \gamma)>0$ also for all $\mu \in[\widetilde{\mu}, 1]$ since $\frac{\partial^{2} \phi_{h}(\mu, \gamma)}{\partial \mu^{2}}<0$, $\phi_{h}(1, \gamma)=0$ and $\left.\frac{\partial \phi_{h}(\mu, \gamma)}{\partial \mu}\right|_{\mu \rightarrow 1}<0$. Therefore $\phi_{h}(\mu, \gamma)>0$ for all $\mu \in[0,1)$ if $\gamma>2$.

Accordingly, $\underline{\delta}^{C}<\underline{\delta}^{h}$ if $\gamma>2$ and $\frac{\theta_{\ell}}{\theta_{h}}=0$. By continuity the same holds for $\frac{\theta_{\ell}}{\theta_{h}} \rightarrow 0$ and $\gamma>2$.

Step 4. Steps 2 and 3 establish that $\underline{\delta}^{C}<\min \left\{\underline{\delta}^{h}, \underline{\delta}^{\ell}\right\}$ if $\gamma>2$ and $\frac{\theta_{\ell}}{\theta_{h}} \rightarrow 0$. Q.E.D.

\subsection{Proof of $\underline{\delta}^{\ell}<\underline{\delta}^{C}$ if $\gamma>2, \frac{\theta_{\ell}}{\theta_{h}} \rightarrow 1$ and $\mu \rightarrow 0$}

Step 1. We derive $\underline{\delta}^{\ell}$ for $\theta_{\ell}=\lambda \theta_{h}$ and $\mu=0$.

Note that $\underline{\delta}^{\ell}=\underline{\delta}^{C}$ if $\theta_{\ell}=\theta_{h}$. Therefore we define $\theta_{\ell}=\lambda \theta_{h}$ and examine $\lambda \rightarrow 1$.

Derive $\underline{\delta}^{\ell}$ by substituting $\theta_{\ell}=\lambda \theta_{h}$ and $\mu=0$ in (A.15) and (A.16). 


$$
\underline{\delta}^{\ell}=\frac{2(\gamma-1) \lambda^{\frac{\gamma}{\gamma-1}}+\gamma(\lambda+1)^{\frac{1}{\gamma-1}}(1-\lambda)-2(\gamma-2)(\lambda+1)^{\frac{\gamma}{\gamma-1}}+(\gamma-1)\left(\frac{1}{2}\right)^{\frac{1}{\gamma-1}}(\lambda+1)^{\frac{\gamma}{\gamma-1}}}{\gamma\left[2\left((\lambda+1)^{\frac{1}{\gamma-1}}-\lambda^{\frac{1}{\gamma-1}}\right)+(\lambda+1)\left((\lambda+1)^{\frac{1}{\gamma-1}}-\left(\frac{1}{2}\right)^{\frac{1}{\gamma-1}}(\lambda+1)^{\frac{1}{\gamma-1}}\right)\right]}
$$

Since $\underline{\delta}^{C}$ is symmetric in $\theta_{\ell}$ and $\theta_{h}$ we can substitute $\alpha=\lambda$ in (43).

$$
\underline{\delta}^{C}=\frac{(\gamma-1)+(\gamma-1) \lambda^{\frac{\gamma}{\gamma-1}}-(\gamma-2)(\lambda+1)^{\frac{\gamma}{\gamma-1}}}{\gamma\left[\left((\lambda+1)^{\frac{1}{\gamma-1}}-\lambda^{\frac{1}{\gamma-1}}\right)+\lambda\left((\lambda+1)^{\frac{1}{\gamma-1}}-1\right)\right]}
$$

Step 2. We show that $\underline{\delta}^{\ell}<\underline{\delta}^{C} \Leftrightarrow \tau_{7}(\lambda, \gamma)<\rho_{7}(\lambda, \gamma)$.

$\underline{\delta}^{\ell}<\underline{\delta}^{C}$ if and only if

$$
\begin{aligned}
& {\left[2(\gamma-1) \lambda^{\frac{\gamma}{\gamma-1}}+(\gamma-1)\left(\frac{1}{2}\right)^{\frac{1}{\gamma-1}}(\lambda+1)^{\frac{\gamma}{\gamma-1}}-2(\lambda+1)^{\frac{\gamma}{\gamma-1}}(\gamma-2)+\gamma(\lambda+1)^{\frac{1}{\gamma-1}}(1-\lambda)\right] } \\
& {\left[\left((\lambda+1)^{\frac{1}{\gamma-1}}-\lambda^{\frac{1}{\gamma-1}}\right)+\lambda\left((\lambda+1)^{\frac{1}{\gamma-1}}-1\right)\right] } \\
< & {\left[2\left((\lambda+1)^{\frac{1}{\gamma-1}}-\lambda^{\frac{1}{\gamma-1}}\right)+(\lambda+1)\left((\lambda+1)^{\frac{1}{\gamma-1}}-\left(\frac{1}{2}\right)^{\frac{1}{\gamma-1}}(\lambda+1)^{\frac{1}{\gamma-1}}\right)\right] } \\
& {\left[(\gamma-1)+(\gamma-1) \lambda^{\frac{\gamma}{\gamma-1}}-(\gamma-2)(\lambda+1)^{\frac{\gamma}{\gamma-1}}\right] }
\end{aligned}
$$

Denote the left-hand-side of equation (A.31) by $\tau_{7}(\lambda, \gamma)$ and the right-hand-side by $\rho_{7}(\lambda, \gamma)$.

Step 3. We show that $\tau_{7}(1, \gamma)=\rho_{7}(1, \gamma)$.

By standard calculations

$$
\tau_{7}(1, \gamma)=\rho_{7}(1, \gamma)=4\left(2^{\frac{1}{\gamma-1}}-1\right)\left[2(\gamma-1)-(\gamma-2) 2^{\frac{\gamma}{\gamma-1}}\right] .
$$

Step 4. We show that $\left.\frac{\partial \tau_{7}(\lambda, \gamma)}{\partial \lambda}\right|_{\lambda=1}>\left.\frac{\partial \rho_{7}(\lambda, \gamma)}{\partial \lambda}\right|_{\lambda=1}$ if $\gamma>2$.

By standard calculations

$$
\begin{gathered}
\left.\frac{\partial \rho_{7}(\lambda, \gamma)}{\partial \lambda}\right|_{\lambda=1}=2^{\frac{\gamma+1}{\gamma-1}}\left[(2-\gamma)+(\gamma-1)\left(\frac{1}{2}\right)^{\frac{1}{\gamma-1}}\right]\left[\frac{3 \gamma+1}{\gamma-1}-\left(\frac{1}{2}\right)^{\frac{1}{\gamma-1}} \frac{3 \gamma+2}{\gamma-1}\right] \\
\left.\frac{\partial \tau_{7}(\lambda, \gamma)}{\partial \lambda}\right|_{\lambda=1}=2^{\frac{\gamma+1}{\gamma-1}} \gamma\left[5\left(\frac{1}{2}\right)^{\frac{1}{\gamma-1}}-\frac{5 \gamma-9}{\gamma-1}\right]\left[1-\left(\frac{1}{2}\right)^{\frac{1}{\gamma-1}}\right]
\end{gathered}
$$

$\left.\frac{\partial \tau_{7}(\lambda, \gamma)}{\partial \lambda}\right|_{\lambda=1}>\left.\frac{\partial \rho_{7}(\lambda, \gamma)}{\partial \lambda}\right|_{\lambda=1}$ if and only if

$$
\begin{aligned}
& 2^{\frac{\gamma+1}{\gamma-1}} \gamma\left[5\left(\frac{1}{2}\right)^{\frac{1}{\gamma-1}}-\frac{5 \gamma-9}{\gamma-1}\right]\left[1-\left(\frac{1}{2}\right)^{\frac{1}{\gamma-1}}\right] \\
> & 2^{\frac{\gamma+1}{\gamma-1}}\left[(2-\gamma)+(\gamma-1)\left(\frac{1}{2}\right)^{\frac{1}{\gamma-1}}\right]\left[\frac{3 \gamma+1}{\gamma-1}-\left(\frac{1}{2}\right)^{\frac{1}{\gamma-1}} \frac{3 \gamma+2}{\gamma-1}\right]
\end{aligned}
$$




$$
\begin{gathered}
\Leftrightarrow-\left(\frac{1}{2}\right)^{\frac{1}{\gamma-1}}\left[2(\gamma-1)\left(\frac{1}{2}\right)^{\frac{1}{\gamma-1}}+\frac{8 \gamma-4 \gamma^{2}-5}{\gamma-1}\right]>2(\gamma-1) \\
\Leftrightarrow 1>2^{\frac{\gamma}{\gamma-1}}(\gamma-1)^{2}\left[1-\left(\frac{1}{2}\right)^{\frac{1}{\gamma-1}}\right]^{2}
\end{gathered}
$$

Denote $\tau_{8}(\gamma)=2^{\frac{\gamma}{\gamma-1}}(\gamma-1)^{2}\left[1-\left(\frac{1}{2}\right)^{\frac{1}{\gamma-1}}\right]^{2}$. Note that $\tau_{8}(2)=1$.

$\tau_{8}^{\prime}(\gamma)=-2^{\frac{\gamma}{\gamma-1}}\left(1-\left(\frac{1}{2}\right)^{\frac{1}{\gamma-1}}\right)\left[\left(1-\left(\frac{1}{2}\right)^{\frac{1}{\gamma-1}}\right) \ln 2-2(\gamma-1)\left(1-\left(\frac{1}{2}\right)^{\frac{1}{\gamma-1}}\right)+2\left(\frac{1}{2}\right)^{\frac{1}{\gamma-1}} \ln 2\right]$

$\tau_{8}^{\prime}(\gamma)<0$ if and only if the term in the square brackets is positive or, equivalently, if and only if

$$
\left(\frac{1}{2}\right)^{\frac{1}{\gamma-1}}[2(\gamma-1)+\ln 2]>(2(\gamma-1)-\ln 2)
$$

Denote $\rho_{9}(\gamma)=\left(\frac{1}{2}\right)^{\frac{1}{\gamma-1}}[2(\gamma-1)+\ln 2]$ and $\tau_{9}(\gamma)=2(\gamma-1)-\ln 2$. It is straightforward to show that

$$
\begin{gathered}
\rho_{9}^{\prime}(\gamma)=\left(\frac{1}{2}\right)^{\frac{1}{\gamma-1}}\left[\frac{1}{(\gamma-1)^{2}}(2(\gamma-1)+\ln 2) \ln 2+2\right]>0, \\
\rho_{9}^{\prime \prime}(\gamma)=\left(\frac{1}{2}\right)^{\frac{1}{\gamma-1}} \frac{1}{(\gamma-1)^{4}}(\ln 2)^{3}>0 .
\end{gathered}
$$

Note that $\rho_{9}(2)>\tau_{9}(2)$ and $\tau_{9}^{\prime}(2)>\rho_{9}^{\prime}(2)$. Furthermore, $\lim _{\gamma \rightarrow \infty} \rho_{9}^{\prime}(\gamma)=\tau_{9}^{\prime}(\gamma)=2$ and $\lim _{\gamma \rightarrow \infty} \rho_{9}(\gamma)=\lim _{\gamma \rightarrow \infty} \tau_{9}(\gamma)$. Since $\rho_{9}(\gamma)$ is convex and $\tau_{9}(\gamma)$ is linear, it follows that $\rho_{9}(\gamma)>$ $\tau_{9}(2)$ for $\gamma>2$ and thus $\tau_{8}^{\prime}(\gamma)<0$ if $\gamma>2$. Therefore, $\tau_{8}(\gamma)<1$ if $\gamma>2$ and, consequently, $\left.\frac{\partial \tau_{7}(\lambda, \gamma)}{\partial \lambda}\right|_{\lambda=1}>\left.\frac{\partial \rho_{7}(\lambda, \gamma)}{\partial \lambda}\right|_{\lambda=1}$ if $\gamma>2$.

Step 5. We have shown that $\tau_{7}(1, \gamma)=\rho_{7}(1, \gamma)$ and $\left.\frac{\partial \tau_{7}(\lambda, \gamma)}{\partial \lambda}\right|_{\lambda=1}>\left.\frac{\partial \rho_{7}(\lambda, \gamma)}{\partial \lambda}\right|_{\lambda=1}$ if $\gamma>2$. Consequently, it follows that $\tau_{7}(\lambda, \gamma)<\rho_{7}(\lambda, \gamma)$ if $\lambda \rightarrow 1$ and $\gamma>2$. Accordingly, $\underline{\delta}^{\ell}<\underline{\delta}^{C}$ if $\gamma>2$, $\frac{\theta_{\ell}}{\theta_{h}} \rightarrow 1$ and $\mu=0$. By continuity the same holds for $\gamma>2, \frac{\theta_{\ell}}{\theta_{h}} \rightarrow 1$ and $\mu \rightarrow 0$. Q.E.D. 